\title{
The Drosophila TRPP Cation Channel, PKD2 and Dmel/ Ced-12 Act in Genetically Distinct Pathways during Apoptotic Cell Clearance
}

\author{
Emeline Van Goethem ${ }^{1 \text { aa }}$, Elizabeth A. Silva ${ }^{1 \text { ab }}$, Hui Xiao ${ }^{2}$, Nathalie C. Franc ${ }^{2 *}$ \\ 1 Medical Research Council Cell Biology Unit, MRC Laboratory for Molecular Cell Biology and Anatomy and Developmental Biology Department, University College \\ London, London, United Kingdom, 2 The Department of Genetics, Affiliated to the Department of Immunology and Microbial Sciences, The Scripps Research Institute, La \\ Jolla, California, United States of America
}

\begin{abstract}
Apoptosis, a genetically programmed cell death, allows for homeostasis and tissue remodelling during development of all multi-cellular organisms. Phagocytes swiftly recognize, engulf and digest apoptotic cells. Yet, to date the molecular mechanisms underlying this phagocytic process are still poorly understood. To delineate the molecular mechanisms of apoptotic cell clearance in Drosophila, we have carried out a deficiency screen and have identified three overlapping phagocytosis-defective mutants, which all delete the fly homologue of the ced-12 gene, known as Dme/lced12. As anticipated, we have found that Dme/lced-12 is required for apoptotic cell clearance, as for its $C$. elegans and mammalian homologues, ced-12 and elmo, respectively. However, the loss of Dmellced-12 did not solely account for the phenotypes of all three deficiencies, as zygotic mutations and germ line clones of Dme/lced-12 exhibited weaker phenotypes. Using a nearby genetically interacting deficiency, we have found that the polycystic kidney disease 2 gene, pkd2, which encodes a member of the TRPP channel family, is also required for phagocytosis of apoptotic cells, thereby demonstrating a novel role for PKD2 in this process. We have also observed genetic interactions between pkd2, simu, drpr, rya-r44F, and retinophilin $(r t p)$, also known as undertaker (uta), a gene encoding a MORN-repeat containing molecule, which we have recently found to be implicated in calcium homeostasis during phagocytosis. However, we have not found any genetic interaction between Dme/lced-12 and simu. Based on these genetic interactions and recent reports demonstrating a role for the mammalian $p k d-2$ gene product in ER calcium release during store-operated calcium entry, we propose that PKD2 functions in the DRPR/RTP pathway to regulate calcium homeostasis during this process. Similarly to its $C$. elegans homologue, Dmel\Ced-12 appears to function in a genetically distinct pathway.
\end{abstract}

Citation: Van Goethem E, Silva EA, Xiao H, Franc NC (2012) The Drosophila TRPP Cation Channel, PKD2 and Dmel/Ced-12 Act in Genetically Distinct Pathways during Apoptotic Cell Clearance. PLoS ONE 7(2): e31488. doi:10.1371/journal.pone.0031488

Editor: Andreas Bergmann, University of Massachusetts Medical School, United States of America

Received June 9, 2011; Accepted January 9, 2012; Published February 8, 2012

Copyright: () 2012 Van Goethem et al. This is an open-access article distributed under the terms of the Creative Commons Attribution License, which permits unrestricted use, distribution, and reproduction in any medium, provided the original author and source are credited.

Funding: This work was funded in part by a Programme Leader career-Track grant from the Medical Research Council United Kingdom to NCF, and Medical Research Council core support to the Medical Research Council Cell Biology Unit. HX is funded by an Irvington Institute Post-doctoral Fellowship from the Cance Research Institute. NCF and her laboratory are funded by The Scripps Research Institute Advanced Discovery Institute start-up grant provided by Novartis to NCF. The funders had no role in study design, data collection and analysis, decision to publish, or preparation of the manuscript.

Competing Interests: NCF and her laboratory are funded by The Scripps Research Institute Advanced Discovery Institute start-up grant provided by Novartis to NCF. This does not alter the authors' adherence to all the PLoS ONE policies on sharing data and materials. NCF and HX are not employed by nor consultants for Novartis. Since the first submission of this article to PLoS One, ES has been recruited by PLoS One as a staff associate editor. There are no patents, products in development, nor marketed products resulting from our research presented in this manuscript.

*E-mail: nfranc@scripps.edu

aa Current address: Institut de Pharmacologie et Biologie Structurale, CNRS UMR 5089, Toulouse, France

ab Current address: Department of Biochemistry and Biophysics, University of California, San Francisco, San Francisco, California, United States of America

\section{Introduction}

During development of all multi-cellular organisms, homeostasis is achieved by controlling cell proliferation and cell death. Apoptosis is a genetically programmed form of cell death that is universally found across phyla $[1,2,3]$. The orderly removal, or phagocytosis, of apoptotic cells is critical in tissue remodelling. Specialized cells or 'professional' phagocytes, such as mammalian macrophages and neutrophils are extremely efficient at engulfing apoptotic cells $[4,5]$. However, 'non-professional' phagocytes may also participate in this process in various tissues [6,7]. Clearance of apoptotic cells generally results in the activation of antiinflammatory signals, and thus may play an important role in the resolution of inflammation in mammals. Indeed it has been proposed that failure to clear apoptotic cells contributes to autoimmune diseases $[8,9,10,11]$, and genes involved in apoptotic cell clearance have mutated splice variants in patients with systemic lupus erythematosus $[12,13]$.

Genetic screens in Caenorhabditis elegans have identified many genes that participate in the clearance of apoptotic cells by neighbouring cells $[14,15,16]$. Among them are the $C$. elegans death genes, ced-1, -2, -5, -6, -7, -10 and -12 , which fall into two partially redundant pathways that converge onto the activation of the Raclike small GTPase CED-10. CED-1, a single transmembrane receptor, acts autonomously in the engulfing cells and clusters around the apoptotic corpse [17]. CED-1 clustering depends on the presence in the engulfing cell of a functional CED-7, an ATPbinding cassette $(\mathrm{ABC})$ transporter also found on the apoptotic cell 
where it was proposed to play a role in apoptotic cell ligand exposure, such as phosphatidylserine (PS) $[17,18]$. CED-6 is a phosphotyrosine binding domain (PTB) containing protein that acts downstream of CED-1, possibly by interacting with its intracellular domain and thus acting as an adaptor protein of CED-1 [19]. CED-2, -5, -10 and -12 were found to act in a parallel and yet partially redundant pathway that controls actin cytoskeleton reorganization in cell corpse engulfment and cell migration [14,15]. CED-2 is an adaptor protein with $\mathrm{SH} 2$ and SH3 domains [20], while CED-5 belongs to the CED-5/ DOCK180/Myoblast city (CDM) family of molecule [21], and CED-12 is a pleckstrin homology $(\mathrm{PH})$ domain-containing protein, which can bind SH3 domains [22,23,24]. CED-12 binds to CED5, which itself physically interacts with CED-2; CED-12 then acts as a bipartite unconventional guanine exchange factor for CED10, as demonstrated for their mammalian homologues, thus highlighting the evolutionary conservation in the molecular mechanisms of this process (reviewed in [14,15]).

In Drosophila, three cell types have been reported to clear apoptotic cells: macrophages (also called plasmatocytes), glial cells and epithelial cells [25,26,27]. We previously reported a role for Croquemort (CRQ), a macrophage receptor related to mammalian CD36, also involved in apoptotic cell clearance [28,29], thereby promoting Drosophila as a good model system in which to genetically dissect the evolutionary conserved molecular mechanisms of phagocytosis of apoptotic cells. Draper (DRPR), another Drosophila receptor with sequence homologies to the $C$. elegans scavenger receptor-related CED-1 also plays a role in phagocytosis of apoptotic cells $[17,30]$. Both DRPR and the Drosophila homologue of CED-6 (Dmel $\backslash$ Ced-6), its adaptor, are also required in glial cells for axon pruning and the engulfment of degenerating neurons [30,31,32]. A Src tyrosine kinase, Src42A, which phosphorylates DRPR and another soluble tyrosine kinase of the Syk family, Shark, which binds to DRPR, were found to be essential for the signalling events downstream of DRPR [33]. Thus far, however, our understanding of the molecular mechanisms underlying phagocytosis of apoptotic cells in this model system has remained sparse.

In search of new genes required for phagocytosis of apoptotic corpses, we have carried out a genetic screen for new Drosophila loss-of-function (LOF) mutants where phagocytosis of apoptotic cells by embryonic macrophages is impaired [34]. We reported a role for Pallbearer (PALL), an F-box protein acting in an E3 ubiquitin ligase complex together with SKPA, a Drosophila Skp1 homologue; LIN19, a Cullin; and Effete (EFF), an E2 ubiquitinconjugating enzyme, in promoting phagocytosis, thus highlighting a novel role for protein degradation via the proteasomal pathway in this process [34]. We recently found that Retinophilin (RTP), also known as Undertaker (UTA), a membrane occupation recognition nexus (MORN) repeat-containing molecule also promotes efficient phagocytosis by regulating $\mathrm{Ca}^{2+}$ homeostasis [35]. We further demonstrated a role for proteins controlling both endoplasmic reticulum (ER) $\mathrm{Ca}^{2+}$-release, such as the Ryanodine Receptor, Rya-r44F, and store-operated $\mathrm{Ca}^{2+}$ entry (SOCE), such as dSTIM and dOrai, in phagocytosis of apoptotic cells and bacteria, thus demonstrating a general role for $\mathrm{Ca}^{2+}$ homeostasis in phagocytosis [35]. Importantly, we found a genetic link between the genes encoding these proteins and both Dme $\backslash$ ced- 6 and $d r p r$, thus highlighting a role for the DRPR pathway in $\mathrm{Ca}^{2+}$ homeostasis during phagocytosis [35].

While Drosophila homologues of CED-2, -5, -10, and -12 exist that include CG1587, Myoblast City (MBC), Rac-2 and Dmel $\backslash$ Ced-12, respectively, their putative role in apoptotic cell clearance has not yet been studied in this model system. Here, we report the phenotypic characterization of three overlapping deficiencies of the
33C-E region of the genome that delete the Drosophila homologue of the ced-12 gene (Dmel \ced-12). Although we found a role for Dme $\backslash$ ced-12 in phagocytosis of apoptotic cells by embryonic macrophages, the deletion of Dmel $\backslash c e d-12$ itself could not account for the phagocytosis-defective phenotype of the deficiencies, as zygotic null mutants of Dmel $\backslash c e d-12$ did not have as strong a phenotype as that of the deficient mutants. This led us to uncover the existence of at least one other gene within this deficiency region that is required for efficient phagocytosis, which we have identified as the Polycystic Kidney Disease 2 TRPP-like cation channelencoding gene, $p k d 2$. We have found a genetic interaction between a null allele of $p k d 2$ and at least one other neighbouring deficiency that also had a strong phagocytosis-defect phenotype. This neighbouring deficiency deletes simu, an EMILIN-like domain containing receptor with homology to CED-1 and DRPR that acts upstream of DRPR in glial cells and binds apoptotic cells [36], and we have further found a genetic interaction between the $p k d 2$ and simu null alleles, arguing that they act in the same pathway. We have also found genetic interactions between the $p k d 2$ null allele and the $r t p$ deficiency, as well as $d r p r$ null and rya-r44F hypomorphic alleles. However, we have found no genetic interaction between a deficiency that deleted Dme $\backslash$ ced-12 (but not pkd2) and the simu deficiency, demonstrating that, as for its C. elegans and mammalian homologues, $D m e l \backslash c e d-12$ is acting in a genetically distinct pathway to that of the DRPR (CED-1) pathway. Thus together our results further support $\mathrm{Ca}^{2+}$ homeostasis as being an integral part of apoptotic cell clearance, and demonstrate that like simu, rtp and rya$r 44 F, p k d 2$ belongs to the DRPR pathway and plays a role in $\mathrm{Ca}^{2+}$ homeostasis during phagocytosis.

\section{Results}

\section{Characterization of overlapping deficiencies of the 33B-E genomic region with phagocytosis defects}

Acridine orange (AO) stains all apoptotic cells in the Drosophila embryo, including those already engulfed by macrophages [37]. When several apoptotic corpses are being engulfed per single macrophage, AO-staining appears in clusters, the distribution pattern of which reflects that of macrophages that have migrated throughout the embryo (figure 1A). We screened the deficiency kit from the Bloomington Drosophila stock center for deletion mutants that lacked AO-stained apoptotic cell-clusters, where macrophages may be phagocytosis-defective in vivo (NCF and K. White, unpublished data). We identified $D f(2 L) p r d 1.7$, $D f(2 L) P r l$, and $D f(2 L)$ esc-P3-0, three large deletions of the 33B-E region of the genome (figure 1D), where AO-stained apoptotic corpses mostly failed to cluster in these homozygous embryos (figure $1 B$ and $G$ and data not shown, respectively), arguing that they may represent novel mutants with defects in phagocytosis of apoptotic cells. In these deficient embryos, apoptotic cells were present at higher levels than in wild-type embryos, and distributed in a 'zebra-like' pattern along the segments of the embryo (compare figures $1 B$ and $C$ with wild-type in figure $\mathbf{1 A}$ ). This apoptosis phenotype coincides with previously reported segmentation defects in these deficiency lines, as homozygous mutant embryos lack 6 out of the 12 segments normally found in wild-type embryos. Indeed, all three deficiencies delete paired (prd), a gene encoding a homeodomain transcription factor of the pair-rule family, which is required for segmentation [38]. With higher levels of apoptosis, we would have expected to observe larger clusters of AO-stained apoptotic cells. Although some large clusters were observed in the heads of these homozygous mutant embryos, we suspected that these zebrapatterns represented cells that are dying in situ without being 

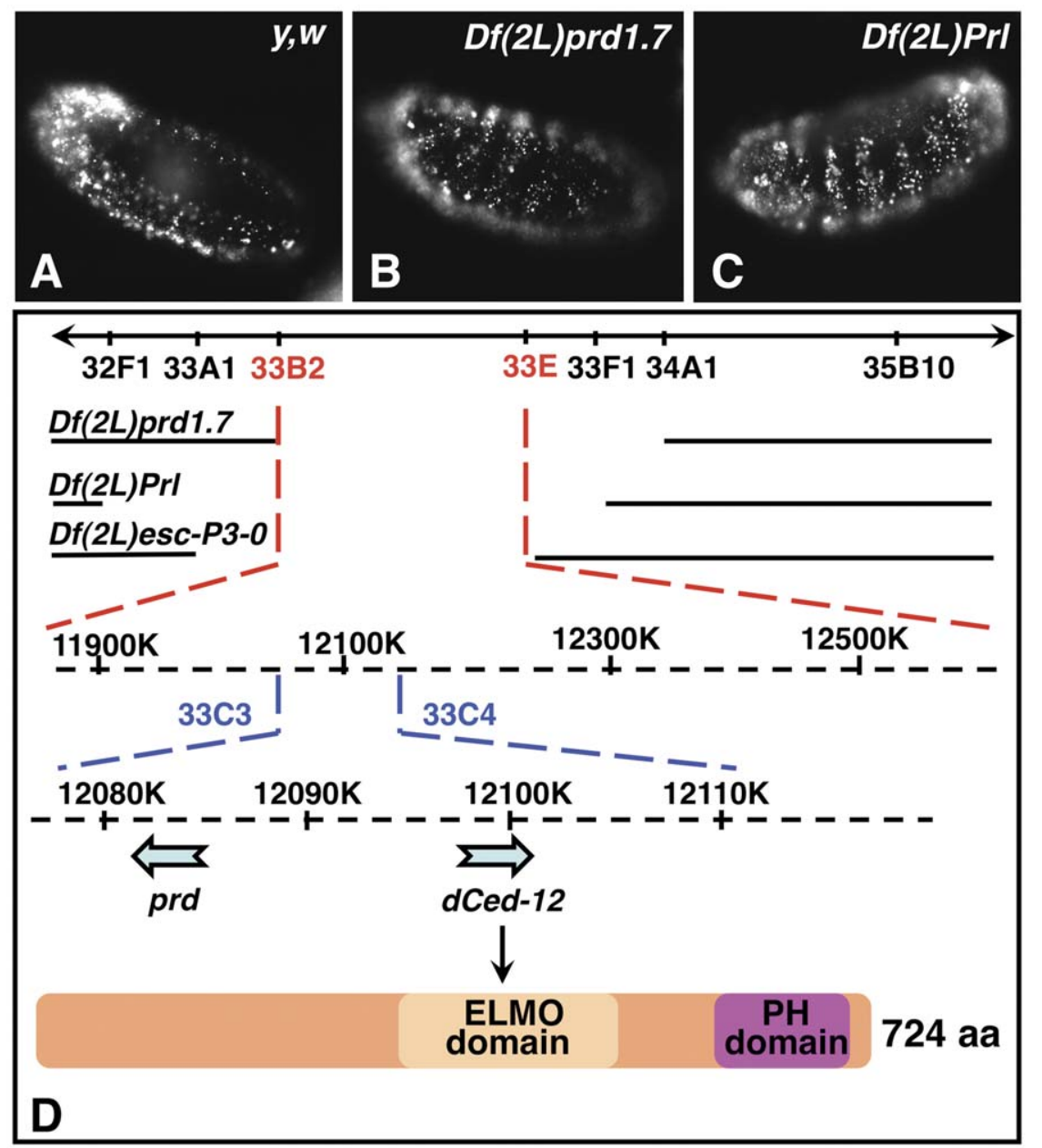

Figure 1. Overlapping deficiencies of the 33B-E genomic region lack clustering of AO-stained apoptotic cells. In A-C, embryos were aged to stage 13, stained with AO, viewed and imaged with a $20 \times$ objective under an epifluorescent Nikon microscope using the red channel, and are presented in a lateral view. A shows a wild-type embryo with a typical pattern of distribution of AO-stained apoptotic cells clusters around the brain lobes and in the posterior end of the embryo. B and C are $D f(2 L) p r d 1.7$ and $D f(2 L) P r l$ homozygous deficient embryos showing increased programmed cell death in a 'zebra-like' pattern along the segments of the embryo. $\mathbf{D}$ is a schematic representation of the 33B-E region of the genome with the breakpoints of all overlapping deficiencies of interest that delete both prd and dced-12. A diagram of the Dmellced-12 protein, with its ELMO and PH domains is also shown.

doi:10.1371/journal.pone.0031488.g001

engulfed by migrating macrophages, resulting from less efficient phagocytosis in these embryos.

The deficiency lines span over $600 \mathrm{~kb}$ and delete about 70 genes, including Dmel $\backslash$ ced-12 or Dmel $\backslash$ elmo, the Drosophila homologue of the $C$. elegans death gene ced-12, and mammalian counterparts elmo-1 and 2 (figure 1D). Similarly to ced-12 and elmo-1 and 2, Dme $\backslash$ ced-12 encodes a protein that contains an ELMO domain (aa 310-490) and a pleckstrin homology (PH) domain (aa 593-673) (figure 1D). Dmel $\backslash$ Ced-12 shares 64, 81 and $79 \%$ of homology with CED-12, ELMO-1 and 2, respectively. CED-12 and ELMO-1 have been shown to be involved in the rearrangement of actin cytoskeleton in apoptotic cell clearance and cell migration $[22,23,24]$. Thus, Dmel $\backslash$ ced-12 is a likely candidate gene to be responsible for the phagocytosis-defect phenotypes observed in all three deficiencies.

Characterization of the phagocytosis phenotypes of candidate deficiencies in the 33B-E genomic region

To further characterize the phenotype of the deficiencies, we stained whole-mount homozygous deficient-embryos with the
GRQ antibody (GRQ Ab), which labels all embryonic macrophages, and 7-amino actinomycin-D (7-AAD), which brightly stains apoptotic nuclei [34]. 7-AAD also stains the nuclei of all cells of the embryo, including that of macrophages, thus allowing us to monitor their ability to engulf apoptotic cells by confocal microscopy by counting the number of macrophages, as well as the number of apoptotic cells they engulf to establish their phagocytic index (PI) (i.e. the mean number of apoptotic cells per macrophage)[34]. As expected, we observed that homozygous embryos for both $D f(2 L) p r d 1.7$ and $D f(2 L) P r l$ deficiencies had segmentation defects that resulted in elevated levels of apoptosis in the region of the missing segments (compare figures $\mathbf{2 B}$ and $\mathbf{C}$ with wild-type in figure $\mathbf{2 A}$ ). The CRQ immunostaining confirmed the presence of macrophages that migrated properly around the brain lobes in the head of wild-type (supplementary figures S1A and S1D), as well as those of $D f(2 L) \operatorname{prd1.7}$ and $D f(2 L) P r l$ homozygous embryos (supplementary figures S1B and $\mathbf{S 1 C}$, respectively). In wild-type embryos, individual macrophages were able to engulf up to 4 corpses (figure 2D), with a mean number of apoptotic cells per macrophage, or 


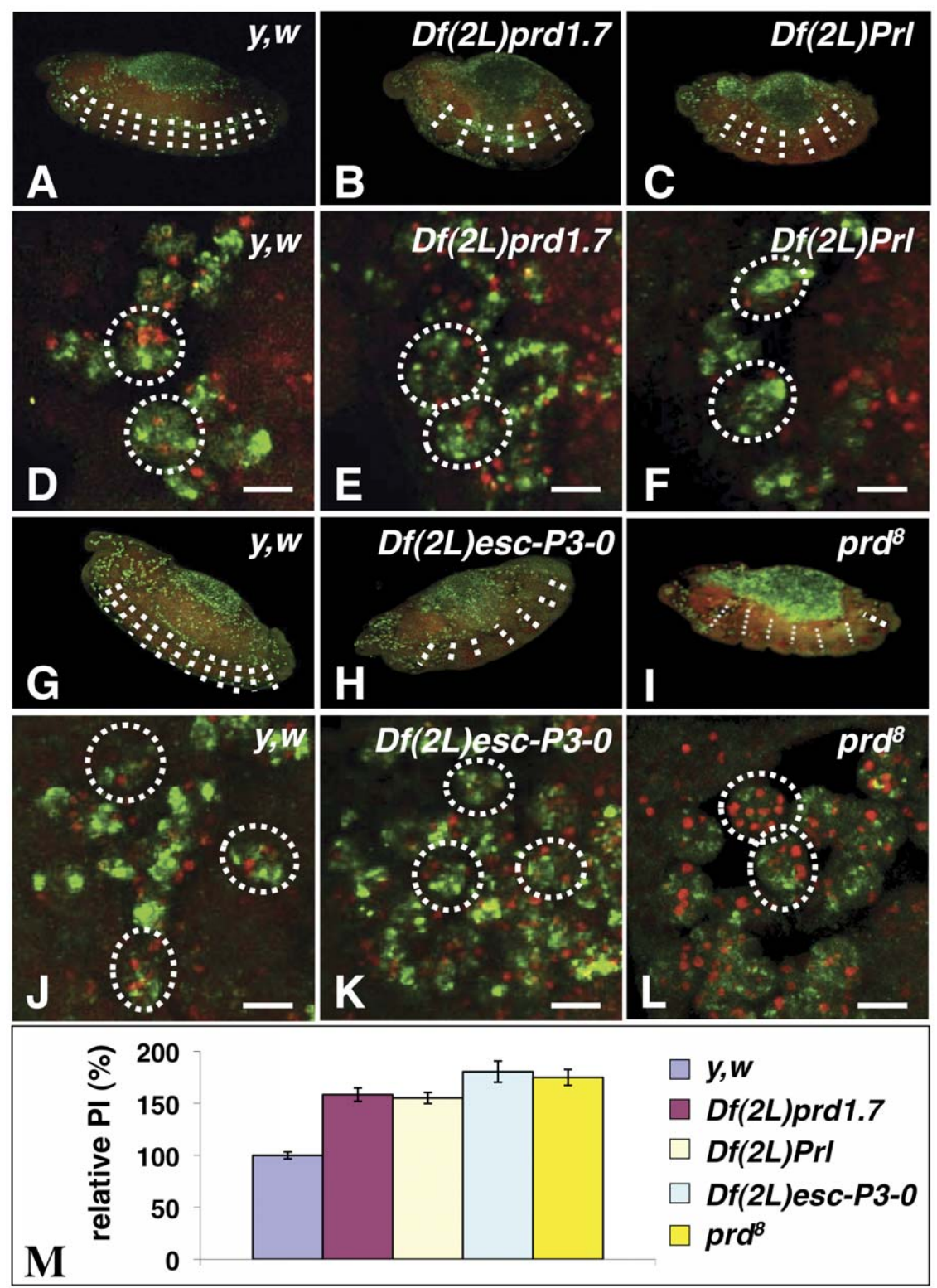

Figure 2. Phenotypic characterization of all three deficiencies and the prd[8] null-allele. In A-L embryos were aged to stage 13 , fixed, their macrophages immunostained with the CRQ Ab (green) and apoptotic corpses detected with 7-AAD staining (red). Confocal images of twelve focal plans taken through macrophages were merged and projected. A-C are $20 \times$ lateral views of a wild-type embryo (A), Df(2L)prd1.7 (B) and Df(2L)Prl (C) homozygous mutant embryos. Homozygous embryos of both $D f(2 L) p r d 1.7$ (B) and $D f(2 L) P r l(C)$ deficiencies show only 6 out of the 12 segments normally observed in wild-type embryos (A), as highlighted by dotted white lines. $\mathbf{D}-\mathbf{F}$ are respective magnified views of macrophages found in the head regions of these embryos, In both homozygous $D f(2 L) p r d 1.7(\mathbf{E})$ and $D f(2 L) P r l(\mathbf{F})$ mutants, macrophages are able of efficiently engulf multiple apoptotic corpses. Scale bars in panels D-F are $5 \mu \mathrm{m}$. G-I are $20 \times$ lateral views of a wild-type embryo (G), Df(2L)Esc-P3-0 (H) and prd[8] (I) homozygous mutant embryos. As expected the deletion of the paired gene causes a segmentation defect in both Df(2L)Esc-P3-0 and prd-null mutant embryos. As seen in $D f(2 L) p r d 1.7(\mathbf{B})$ and $D f(2 L) P r l(\mathbf{C}), D f(2 L) E s c-P 3-0(\mathbf{H})$ and $\operatorname{prd}[8](\mathbf{I})$ homozygous mutant embryos also have only 6 out of the 12 segments normally observed in wild-type embryos (A and $\mathbf{G})$ due to their loss of prd function. $\mathbf{J}-\mathbf{L}$ are higher magnification views of macrophages within the head of $D f(2 L) E s c-P 3-0$ and prd[8] homozygous mutant embryos, respectively. Mutant macrophages in both $D f(2 L) E s c-P 3-O$ (K) and prd-null (L) homozygous embryos efficiently engulf multiple apoptotic corpses, with prd-null macrophages occasionally engulfing up to 12 corpses. Scale bars in panels $\mathbf{J}-\mathbf{L}$ are $5 \mu \mathrm{m}$. Phagocytic indices for wild-type, the deficiencies, and the prd-null mutant embryos have been quantified and are summarised in a graph in $\mathbf{M}$. Pls calculated from three image-stacks taken from 5 to 15 embryos of each genotype were normalized against wild type (with wildtype relative PI set as 100\%) and are presented as relative PIs \pm standard error of the mean (SEM) for each genotype. In D-F, and J-L, dotted white circles are indicative of individual macrophage cell bodies based on 7-AAD staining of their regular nuclei and CRQ staining. doi:10.1371/journal.pone.0031488.g002

phagocytic index (PI) of $1.89 \pm 0.06$ (normalized to $100 \% \pm 3.3$ in figure 2M). Surprisingly, and seemingly in contrast with our previous observation that $\mathrm{AO}$-stained apoptotic cells appeared to mostly fail to cluster in these embryos, arguing that their macrophages may be phagocytosis-defective, we observed that
$D f(2 L) \operatorname{prd} 1.7$ and $D f(2 L) \operatorname{Prl}$ homozygous macrophages were able to efficiently engulf multiple corpses (figures $2 \mathbf{E}$ and $\mathbf{F}$, respectively). When compared to wild type macrophages, for which we normalized the PI to $100 \%, D f(2 L) p r d 1.7$ and $D f(2 L) \operatorname{Prl}$ homozygous macrophages showed a $58 \pm 6$ and $55 \pm 5 \%$ increase in their 
relative PIs, respectively ( $\mathrm{p}$ values $<0.0001)$ (figure $\mathbf{2 M}$ ). As in $D f(2 L) p r d 1.7$ and $D f(2 L) P r l$, homozygous embryos for $D f(2 L) e s c-P 3-$ 0 , a third overlapping $D m e l \backslash c e d-12$ deletion, also had a severe segmentation defect associated with the loss of prd, with only six segments present (figure 2H). $D f(2 L)$ esc-P3-0 homozygous macrophages also did not appear to show any major defect in migration (Supplementary figure S1E) or phagocytosis of apoptotic cells (compare figure $2 \mathrm{~K}$ with wild type macrophages in 2J), as they engulfed more apoptotic cells than wildtype macrophages with an $80.5 \pm 10 \%$ increase in PI (p value $<0.0001)$ (figure $\mathbf{2} \mathbf{M}$ ).

We suspected that these results might reflect the presence of elevated levels of apoptosis observed in these mutant embryos by comparison to wild types. Although these mutant macrophages may be less efficient at engulfing apoptotic cells, as suggested by the lack of AO-stained apoptotic cell-clusters in the deficient-embryos, their phagocytosis phenotype might be masked by the excess of apoptotic cells present. In order to separate the cell death defect from potential engulfment defects, we examined the phagocytic ability of macrophages within $p r d^{8}$ LOF mutant embryos, as well as the pattern of distribution of apoptotic cells in these embryos. As expected, $p r d^{o}$ LOF mutant embryos had only six segments due to the lack of PRD function resulting in a segmentation defect (figure 2I). As in all three deficient lines, $p r d^{g}$ mutant macrophages also migrated properly in the head region (supplementary figure S1F) and engulfed significantly more apoptotic corpses than wild-type macrophages (compare figures $2 \mathbf{L}$ with $2 \mathbf{J}$, and supplementary figure S2B with S2A), with a $75 \pm 8 \%$ increase in their relative PI (figure $\mathbf{2 M})(\mathrm{p}<0.001)$. These results were in keeping with the elevated levels of apoptosis due to the $p r d^{8} \mathrm{LOF}$ mutation-associated segmentation defect.

Surprisingly, however, we found that the pattern of distribution of apoptotic cells in $p r d^{g}$ mutant embryos differed from that of each of the three deficiencies, as determined by Terminal deoxyribonucleotide transferase (TdT)-mediated dUTP Nick End Labelling (TUNEL). Indeed, while all mutant embryos had increased levels of apoptosis when compared to wild-type embryos (compare figures 3B and 3D-F with figure $\mathbf{3 A}$ ), $p r d^{g}$ mutant embryos appeared to have fewer apoptotic cells than each of the three deficiencies. More importantly, we observed that TUNEL-stained apoptotic cells in $p r d^{g} \mathrm{LOF}$ were found in large clusters (figure 3G and inset). Instead, and as seen previously in their AO-staining (see figure 1), $D f(2 L) p r d 1.7, D f(2 L) P r l$ and $D f(2 L) e s c-P 3-0$ homozygous mutants had excessive amounts of apoptotic cells in a "zebra-like" distribution pattern (figure 3D, E and F, respectively). Thus, while some clustering of TUNEL-stained apoptotic cells could still be observed in these deficiency-mutant embryos (see inset in figure 3H), and while their PIs indicated
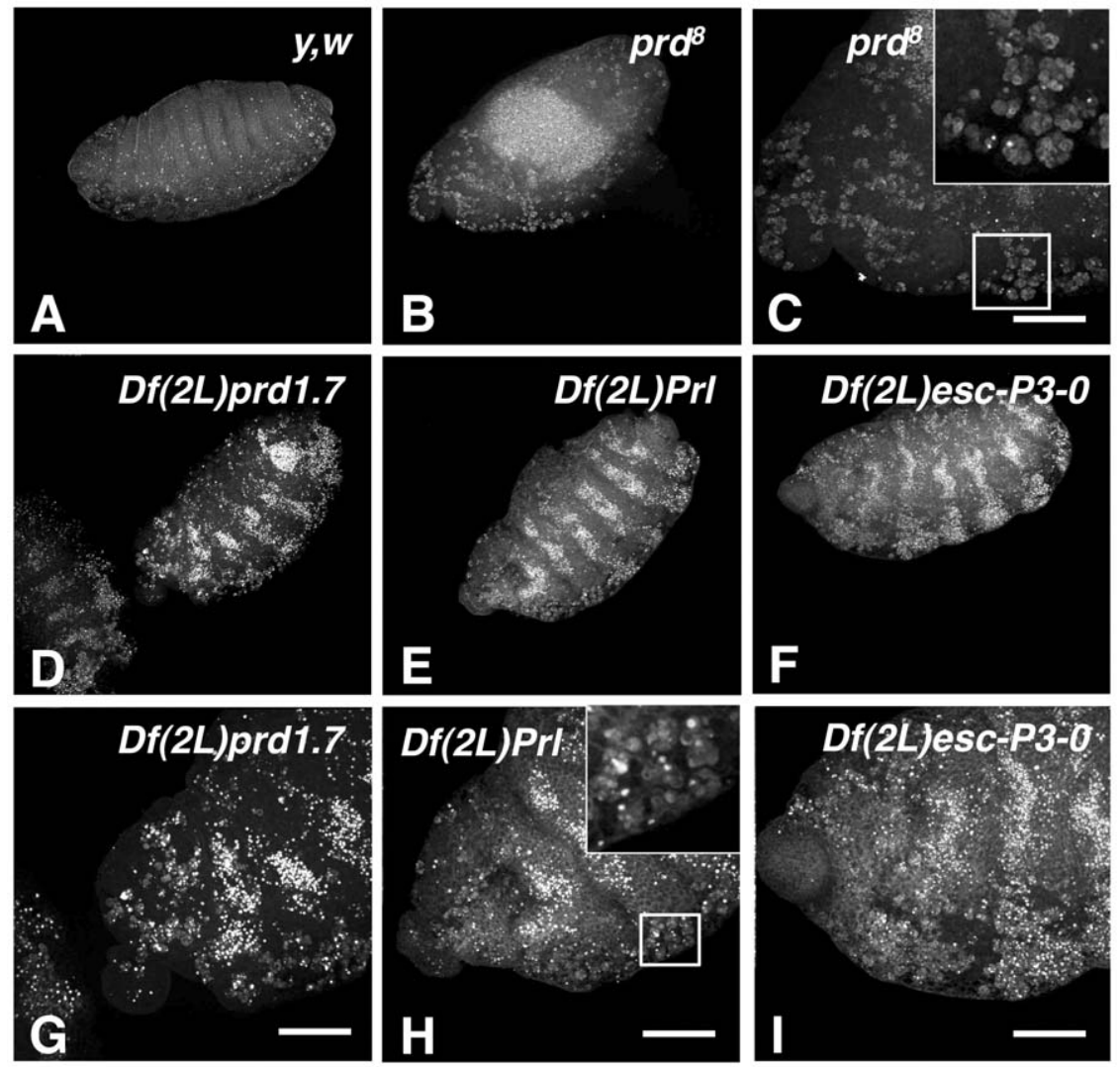

Figure 3. TUNEL-staining pattern argue that mutant macrophages in all three dced-12-deficiencies fail to efficiently clear apoptotic cells. A-I are projected confocal images of stage 13 embryos subjected to TUNEL. In $\mathbf{A}$ is a wild-type embryo where labelled apoptotic cells are found throughout the embryo in small clusters reflecting their engulfment by macrophages. In $\mathbf{B}$ and $\mathbf{C}$, a $20 \times$ and enlarged views of a prd[8]-null mutant are shown where, as expected, TUNEL-labelled apoptotic cells are present at a higher level, with big clusters of labelled apoptotic cells seen that reflect their engulfment by prd[8] macrophages. In TUNEL of $D f(2 L)$ prd1.7 (D), Df(2L)Prl (E) and Df(2L)esc-P3-0 (F) homozygous embryos, a cell death pattern in stripes is observed along the segments, arguing that macrophages in these deficiencies are not able to clear apoptotic cells as efficiently as macrophages do in prd[8]-null mutants. G-I are $40 \times$ magnified counterparts of $\mathbf{D}-\mathbf{F}$, where some clusters of labelled apoptotic cells can still be observed (arrows), although the majority of labelled apoptotic cells remain scattered around the embryo. Scale bars in panels G-I are $50 \mu \mathrm{m}$. doi:10.1371/journal.pone.0031488.g003 
that their macrophages were capable of engulfing multiple apoptotic cells, their patterns of distribution of AO or TUNEL stained-apoptotic cells argue that they are less efficient than $p r d^{8}$ macrophages in clearing the vast amount of apoptotic cells generated during defective segmentation.

Dme/lced-12 is required for efficient engulfment of apoptotic corpses by macrophages, but does not solely account for the phagocytosis-defect observed in Df(2L)prd1.7 mutant embryos

To assess the efficiency of $D f(2 L) p r d 1.7$ deficient macrophages in the presence of wild-type levels of apoptosis, we rescued the apoptotic cell-death associated with the prd deletion in the
$D f(2 L)$ prd1.7 background using a previously characterized prd genomic rescue transgene [38]. These prd-rescued $D f(2 L)$ prd1.7 macrophages were less efficient in clearing apoptotic cells than that of wild type embryos (compare figure 4B with wild-type in figure $4 \mathbf{A})$ with a $52 \%$ decrease in their PI $(\mathrm{p}<0.001)$ (figure 4G). As a consequence of their failure to efficiently engulf apoptotic cells, prd-rescued $D f(2 L)$ prd1.7 mutant macrophages appeared smaller in size than wild-type macrophages (compare figure 4B with figure 4A), as previously described in other phagocytosis-defective mutant macrophages, such as those of $\mathrm{crq}$-deficient and pallbearer (pall) LOF mutants [29,34]. Thus, these results confirm that $D f(2 L)$ prd1.7 deletes at least one gene required for efficient phagocytosis of apoptotic corpses.
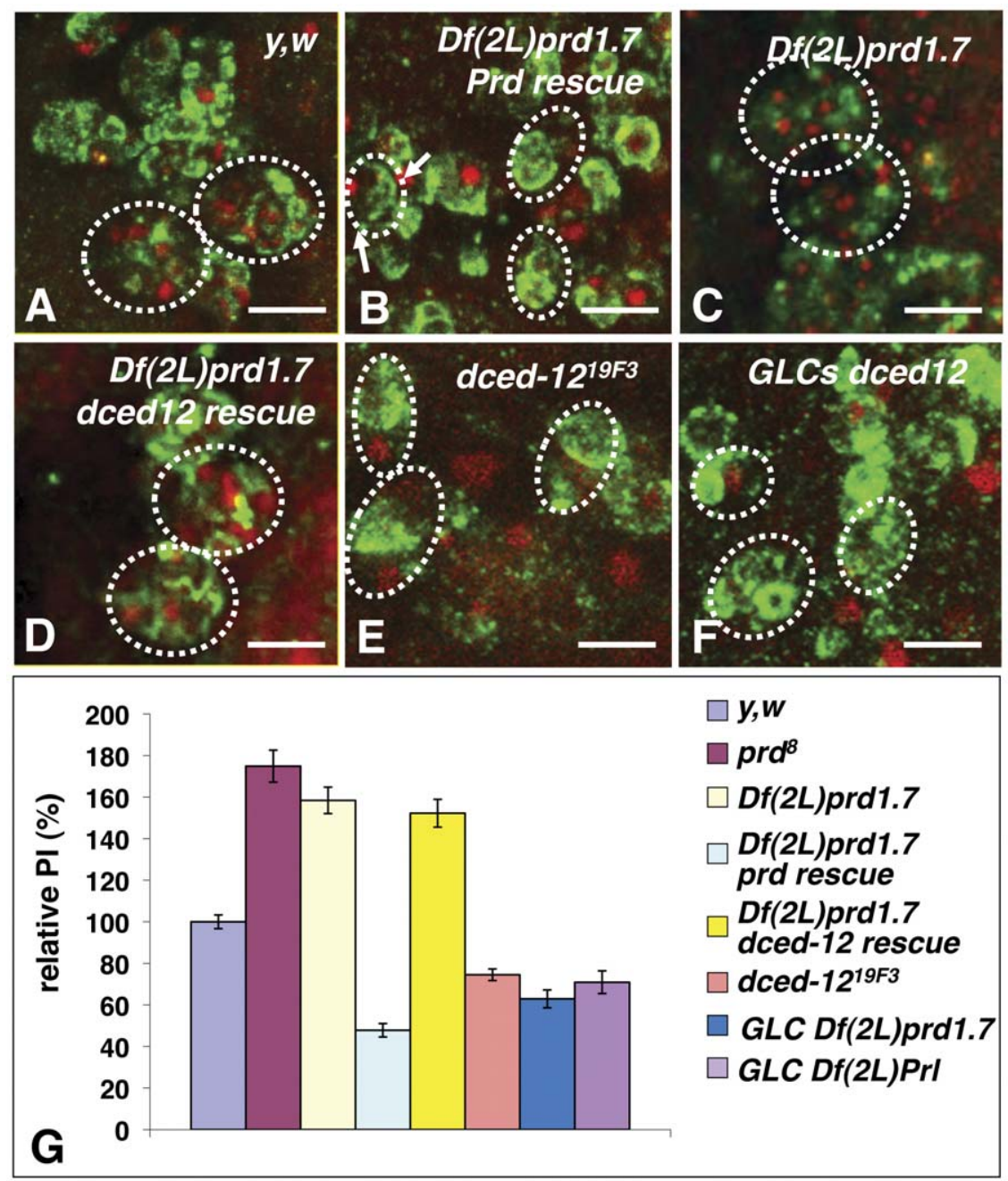

Figure 4. Dme/lced-12 is required in macrophages for efficient clearance of apoptotic cells. In A-F are projected confocal images of macrophages of stage 13 embryos stained with the anti-CRQ Ab (green) and where apoptotic corpses are stained with 7-AAD (red). In A, wild-type macrophages are shown that appear large as they engulf several apoptotic cells each. In B, prd-rescued Df(2L)prd1.7 homozygous mutant macrophages fail to efficiently engulf multiple corpses and appear small. $D f(2 L) p r d 1.7$ homozygous mutant macrophages where a UAS-Dme/lced12 transgene is being specifically over-expressed using a crq-gal4 driver on the third chromosome (D) do not engulf significantly more efficiently than in absence of the UAS-Dme/lced-12transgene (C). In E and F are images of macrophages within the head of dced-12[19F3] homozygous mutant embryos (D) and that of macrophages of dced-12[PB] GLCs in trans- with Df(2L)prd1.7 (F). Macrophages in dced-12[19F3] and GLCs mutant embryos can still engulf apoptotic cells, albeit at a lower efficiency than that of wild-types $(\mathbf{A})$. Scale bars in $\mathbf{A}-\mathbf{F}$ are $5 \mu \mathrm{m}$. A graph in $\mathbf{G}$ summarizes the quantification of the Pls of wild-type (yw), $D f(2 L) p r d 1.7$, prd-rescued $D f(2 L)$ prd1.7, dced-12-rescued $D f(2 L) p r d 1.7$ on the third chromosome, dced12[19F3], and Dmellced-12GLCs homozygous macrophages. Each bar represents the mean value \pm SEM of the relative PIs for each genotype. Scale bars in panels $\mathbf{A}-\mathbf{F}$ are $5 \mu \mathrm{m}$. In A-F, dotted white circles are indicative of individual macrophage cell bodies based on 7-AAD staining of their regular nuclei and $C R Q$ staining.

doi:10.1371/journal.pone.0031488.g004 
To address whether the phagocytosis defect might be associated with the LOF of Dmel $\backslash c e d-12$, we carried out a Dmel $\backslash c e d-12$ rescue experiment. We generated a $U A S-D m e \backslash \backslash$ ced-12 transgenic line on the $\mathrm{X}$ chromosome and established a fly line carrying both $D f(2 L)$ prd1.7 and the UAS-Dmel $\backslash c e d-12$ transgenes. A $D f(2 L)$ prd1.7 deficiency line was also established that carried a $\mathrm{crq}_{\text {-gal4 }}$ transgene on the third chromosome (a kind gift of Drs N. Perrimon and H. Agaisse). By crossing the deficiency line carrying the UAS-Dmel $\backslash$ ced-12 transgene to the deficiency line carrying the $c r q$-gal4 driver, we were able to specifically drive the expression of Dmel $\backslash$ ced-12 in $D f(2 L)$ prd1.7 homozygous macrophages. We found that re-expressing Dmel $\backslash$ ced12 was not sufficient to significantly increase the ability of Df(2L)prd1.7 mutant macrophages to clear apoptotic cells (compare figures $4 G$ and $D$ ), as their PI was strikingly similar to that of $D f(2 L) p r d 1.7$ homozygous macrophages alone (relative $\mathrm{PI}=152.2 \pm 6.7 \%$ versus $158.4 \pm 6.4 \%, \mathrm{p}>0.05)$ (figure 4G). Thus, re-expressing Dmel $\backslash$ ced-12 does not appear to significantly increase the ability of $D f(2 L) p r d 1.7$ macrophages to efficiently engulf apoptotic cells, arguing against the possibility that Dmel $\backslash$ ced-12 is the only gene responsible for this deficiency phenotype.

As demonstrated previously, a possibility remained that the excessive number of apoptotic cells present in $D f(2 L)$ prd1.7 embryos and the consequently elevated PI of $D f(2 L) \operatorname{prd} 1.7$ macrophages might mask the effect of a $D m e l \backslash c e d-12$ rescue. We could not establish the necessary lines to carry a double-rescue experiment of $D f(2 L)$ prd1.7 mutant embryos with both prd and Dmel $\backslash$ ced-12 transgenes; therefore we assessed the phagocytosis phenotype of homozygous embryos carrying a single $D m e l \backslash c e d-12$ null allele, ced$12^{19 F 3}[39]$ (figure 4E). We found that ced-12 ${ }^{19 F 3}$ mutant macrophages were significantly less efficient than wild-type macrophages in clearing apoptotic cells with a $25.5 \pm 2.8 \%$ decrease in their PIs (PI $=74.5 \% \pm 2.8 \%$, and $\mathrm{p}<0.001$ )(figure 4G). The PI of $c e d-12^{19 F 3}$ macrophages, however, was significantly higher than that of prd-rescued $D f(2 L)$ prd1.7 macrophages, which had a PI of $47.8 \% \pm 3.2 \%(\mathrm{p}<0.001)$ (figure $4 \mathbf{G})$, arguing that Dmel $\backslash$ ced- 12 may not be the sole gene responsible for the phenotype seen in the deficiency embryos.

We, and others, have observed a strong maternal contribution of Dme $\backslash$ ced -12 mRNA in early embryos that may compensate for the zygotic LOF phenotype of $D m e l \backslash c e d-12$ (data not shown and $[39,40])$. Therefore we also analyzed $D m e \backslash \backslash$ ced-12 germ line clones (GLCs). We were not able to recover any GLCs with the ced-12 $19 F 3$ null allele in combination with either Dmel $\backslash c e d-12$ deficiency. Thus, GLCis were generated with a hypomorphic allele of Dmel\ced-12, ced$12^{P B 06760}$, in combination with either $D f(2 L) \operatorname{prd} 1.7$ (figure 4G) or $D f(2 L) p r l$ (figures $\mathbf{4 F}$ and $\mathbf{G})$. In these embryos, the maternal germ line genotype is homozygous for ced-12 $12^{P B 06760}$, and their zygotic genotype is heterozygous for ced-12 $2^{P B 06760}$ and either $D f(2 L) \operatorname{prd} 1.7$, or $D f(2 L)$ prl. We examined their phagocytosis phenotypes and observed that macrophages in these mutant embryos had a similar phenotype to that of $c e d-12^{19 F 3}$ zygotic null mutant macrophages with relative PIs of $62.9 \pm 4.3 \%$, and $70.9 \pm 5.5$, respectively, versus $74.5 \pm 2.8$ (with $\mathrm{p}$ values $>0.05$ )(figure 4G). Thus, in a similar wildtype context of apoptosis, the PIs of ced-12 $19 F 3$ and ced-12 $2^{P B 06760}$ GLCs macrophages were lower than that of wild-type macrophages $(\mathrm{p}<0.001)$, arguing in favour of a role for Dmel $\backslash$ ced-12 in phagocytosis of apoptotic cells. However, our observation that the PIs of dced-12 $2^{19 F 3}$ homozygous mutant macrophages and of Dmel $\backslash c e d-12$ GLCs were significantly higher than that of $p r d$-rescued $D f(2 L)$ prd1.7 macrophages, demonstrated that Dmel $\backslash$ ced-12 was only partially responsible for the phenotype observed in prd-rescued $D f(2 L)$ prd1.7 mutant embryos $(\mathrm{p}<0.05)$ (figure 4G). Altogether these results show that, as for elmo in mammals and ced-12 in $C$. elegans, Dmel $\backslash$ ced-12 plays a role in phagocytosis of apoptotic cells in the fly; these data also argue in favour of the presence of at least one other gene in the $D f(2 L)$ prd1.7 deficiency that is required for phagocytosis. We will hereafter refer to this gene as gene $X$.

\section{Genetic interactions with $D f(2 L) b 87 e 25$ reveal the} presence of at least one novel gene required for phagocytosis of apoptotic cells in the 33E-F1 genomic region

In order to narrow down the region where gene $X$ may be positioned, we screened additional overlapping and nearby deletions of the 33-34 region of the genome (figure 5A). We found that $D f(2 L) b 87 e 25$ homozygous embryos also had phagocytosis-defective macrophages similar to that of prd-rescued $D f(2 L)$ prd1.7 embryos, with a relative PI of $54.6 \pm 3.0 \%$ versus $47.8 \pm 3.2 \%$, respectively (figure 5B, and 5E) $(\mathrm{p}>0.05)$. Single embryo mapping of the breakpoints of this novel phagocytosis-defective deficiency mutant confirmed that it deleted the 34B12-35B10 region of the genome, thus revealing the presence of yet another gene of interest in this region that is required for phagocytosis of apoptotic cells (figure 5A). Interestingly, a gene required for phagocytosis of apoptotic cells by glial cells, six microns under (simu) was characterized by Dr. E. Kurant and colleagues that is deleted in the $D f(2 L) b 87 e 25$ mutant line [36] and is most likely responsible for the defect we observed in these Df $(2 L) b 87 e 25$ homozygous embryos.

This 34B12-35B10 genomic region, however, does not overlap with either region of the previous three deficiencies. Thus, to test for any potential genetic interactions between these lines, we next assessed the phagocytosis-defective phenotypes in heterozygous mutant combinations between $D f(2 L) b 87 e 25$ and $D f(2 L)$ prd1.7, $D f(2 L) \operatorname{Prl}$ or $D f(2 L)$ esc-P3-0. All heterozygous mutant combinations were wild type for segmentation and consequently showed wild-type levels of apoptosis. Thus their PIs could be compared to that of prdrescued $D f(2 L) \operatorname{prd1.7}$ macrophages. We found that heterozygous macrophages for $D f(2 L) b 87 e 25$ and either $D f(2 L) p r d 1.7$ or $D f(2 L) P r l$ were phagocytosis-defective (data not shown and figure $\mathbf{5 C}$, respectively), and had relative PIs of $62.5 \pm 6.6 \%$ and $55.8 \pm 4.6 \%$, respectively, which were similar to that of $p r d$-rescued $D f(2 L)$ prd1.7 macrophages with a relative PI of $47.8 \pm 3.2 \%$ (figure 5E)(with $\mathrm{p}$ values $>0.05)$. Embryos heterozygous for $D f(2 L) b 87 e 25$ and $D f(2 L)$ esc-P3-0, however, appeared wild-type for apoptosis and phagocytosis with a PI of $105 \pm 6.7 \%$ (figures 5D, and 5E) $(\mathrm{p}>0.05$ when compared to wild type, and $\mathrm{p}<0.001$ when compared to prdrescued $D f(2 L) \operatorname{prd1}$.7). These results demonstrate a genetic interaction between at least one gene within the deficiency region of $D f(2 L) b 87 e 25$, which is likely to be simu, and gene $X$. These also allow us to localize gene $\mathrm{X}$ between the proximal breakpoints of $D f(2 L)$ escP3- 0 and $D f(2 L) P r l$, i.e. within the $33 \mathrm{E}$ to $33 \mathrm{~F} 1$ region of the genome (see yellow brackets in figure 5A). This localization of gene $X$ is in agreement with our previously calculated PIs showing that $D f(2 L)$ esc-P3-0 macrophages engulfed slightly but significantly more apoptotic cells than $D f(2 L)$ prl macrophages, with a relative PI of $180.5 \pm 10.2 \%$ versus PIs of $155.2 \pm 5.3 \%(\mathrm{p}<0.05)$, while there was no significant difference between $D f(2 L)$ prd1.7 and $D f(2 L)$ prl PIs $(\mathrm{p}>0.05)$ (figure 2M). These genetic interactions refined the region associated with gene $X$ to about $306 \mathrm{~kb}$ containing 22 predicted genes. Finally, these results also argue that there are no genetic interactions between the gene(s) responsible for the phenotype observed in $D f(2 L) b 87 e 25$ homozygous mutants and Dmel`ced-12.

$p k d 2$ is required for apoptotic cell clearance and genetically interacts with rya-r44F, rtp, drpr and simu, but simu does not interact with Dme/lced-12

Among the 22 genes of the 33E-F1 region is pkd2, the polycystic kidney disease 2 gene ( $p k d 2)$, also known as almost there (amo). pkd2 is a 


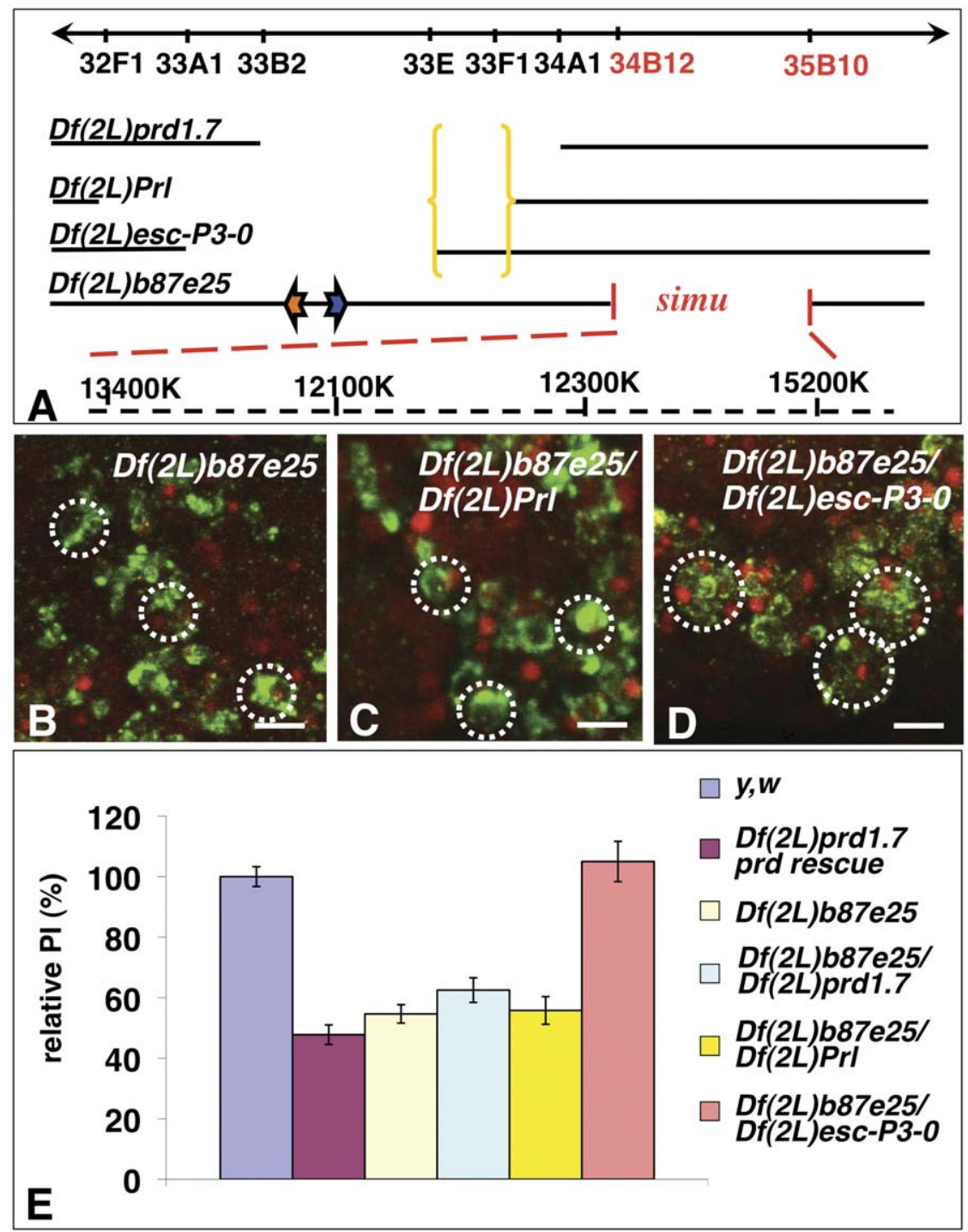

Figure 5. Genetic interactions with an adjacent deficiency of the 33C-E region reveals the existence of at least one novel gene required for phagocytosis of apoptotic cells in the 33E-F1 region. A is a schematic representation of the 33B-E region of the genome with the breakpoints of all overlapping deficiencies of interest that delete both prd and dced-12, and $D f(2 L) b 87 e 25$, a neighbouring deficiency. The prd gene is represented as an orange arrow, and Dmel/ced-12 as a blue arrow. In B-D are projected confocal images of macrophages in stage 13 Df(2L)b87e25 homozygous deficient embryos (B) and trans-heterozygous $D f(2 L) b 87 e 25 / D f(2 L) p r d 1.7(\mathbf{C})$ and $D f(2 L) b 87 e 25 / D f(2 L) e s c-P 3-0$ (D) that were stained with the CRQ Ab (green) and 7-AAD (red). Macrophages of both $D f(2 L) b 87 e 25$ homozygous and Df(2L)b87e25/Df(2L)Prl trans-heterozygous embryos are smaller in size and generally less efficient at engulfing apoptotic cells than that of $D f(2 L) b 87 e 25 / D f(2 L)$ esc-P3-0 that appear larger with several apoptotic corpses within them. These results demonstrate the presence of at least one gene in the 33E-F1 genomic region that is required for phagocytosis of apoptotic cells (see yellow brackets in A). In E is a graph summarizing the Pls of wild-type $(y, w)$, prd-rescued $D f(2 L) p r d 1.7$, Df(2L)b87e25, homozygous macrophages and Df(2L)b87e25/Df(2L)prd1.7, Df(2L)b87e25/Df(2L)Prl, Df(2L)b87e25/Df(2L)esc-P3-0. Each bar represents the mean value \pm SEM of the relative Pls for each genotype. Scale bars in panels B-D are $5 \mu \mathrm{m}$. In B-D, dotted white circles are indicative of individual macrophage cell bodies based on 7-AAD staining of their regular nuclei and CRQ staining.

doi:10.1371/journal.pone.0031488.g005

homologue of the mammalian polycystin-2 gene, which encodes PC2, a Ca ${ }^{2+}$-activated $\mathrm{Ca}^{2+}$ permeable cation channel with distant homologies to TRPP family of cation channels [41]. Drosophila pkd2 or amo is required for directional sperm movement and male fertility $[42,43]$. In combination with a mutation of the ryanodine receptor gene, rya-r44F, which encodes a $\mathrm{Ca}^{2+}$ channel on the endoplasmic reticulum (ER), a mutation in $p k d 2$ also lowers the rate of cell body wall contraction in Drosophila larvae [41]. Interestingly, we recently found a role for Rya-r44F, Retinophilin (RTP) (also known as UTA), a membrane occupation nexus repeat (MORN)-containing protein, and for molecules that promote store-operated $\underline{\mathrm{Ca}}^{2+}$ entry (SOCE) in phagocytosis of apoptotic cells and bacteria where they regulate $\mathrm{Ca}^{2+}$ homeostasis [35]. pkd2 may therefore be in the same genetic pathway as $r t p$ and $r y a-r 44 F$ in controlling $\mathrm{Ca}^{2+}$ homeostasis during phagocytosis.

We asked whether pkd2 might be required for phagocytosis of apoptotic cells in vivo by assessing the phagocytosis phenotype of a previously generated knock-out allele of $p k d 2$, amo ${ }^{1}$ [43]. We found that homozygous mutant macrophages for $a m 0^{1}$ were less efficient at engulfing apoptotic cells than wild-type macrophages (compare figures $\mathbf{6 A}$ and $\mathbf{B})$ with a relative PI of $51.7 \pm 2.6 \%$ $(\mathrm{p}<0.001)$ (figure 6G), thereby confirming a role for $p k d 2$ in 

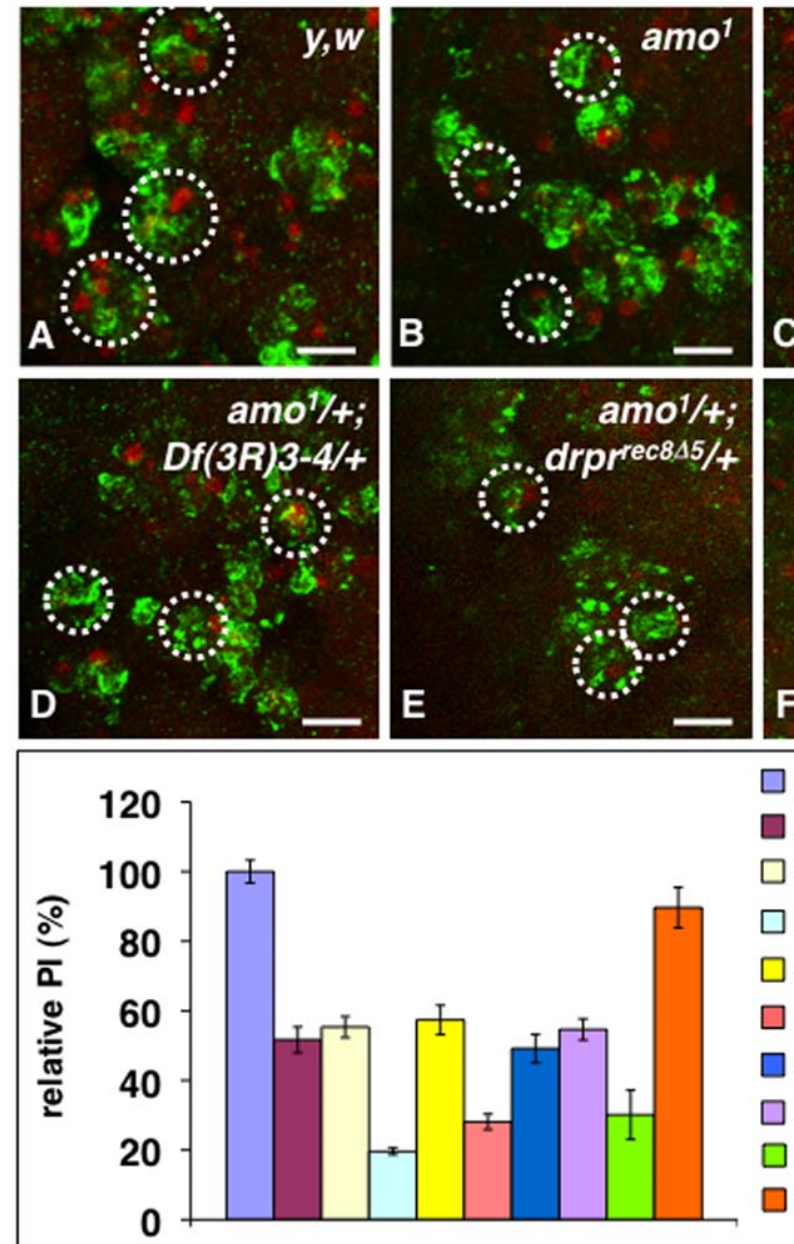

\section{$\square y, w$}

$\square a^{1} o^{1}$

$a m o^{1 / t+;} D f(3 R) 3-4 /+$

$D f(3 R) 3-4$

amo $1 /+$; drprec845/+

drprec845

$a^{2} o^{1 / D f(2 L) b 87 e 25}$

Df(2L)b87e25

amo1/simu2

ced-12 19 FF $/$ simu $^{2}$

G

Figure 6. Genetic interactions between $p k d 2$, uta and drpr null alleles reveal a role for $p k d 2$ in phagocytosis of apoptotic cells. In A-F are projected confocal images of macrophages in stage $13 y, w$ (wild-type) embryos (A), amo ${ }^{1}$ homozygous embryos (B), amo $/$ rya-r44 $^{k 04913}$ heterozygous embryos (C), double heterozygous $a m o^{1} /+; D f(3 R) 3-4 /+$ (D) and $a m o^{1} /+; d r p r^{r e c} 45 /+$ (E) embryos, and $a m o^{1} / D f(2 L) b 87 e 25$ heterozygous $^{2}$ embryos (F). All embryos were stained with the CRQ Ab (green) and 7-AAD (red). Embryos in B-F had macrophages that were smaller and less efficient at engulfing apoptotic cells than that of wild-type embryos (A). In $\mathbf{G}$ is a graph summarizing the Pls of wild-type $(y, w), a m o 1, r y a-r 44 F^{k 04913}$,

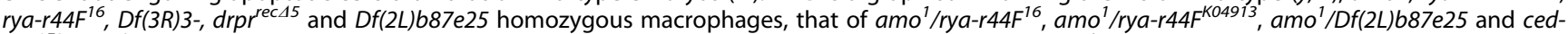
$12^{19 F 3} /$ simu $^{2}$ trans-heterozygous macrophages, as well as that of $a m o^{1} /+; D f(3 R) 3-4 /+$ and $a m^{1} /+; d r r^{r e c} 45 /+$ double heterozygous macrophages. Each bar represents the mean value \pm SEM of the relative Pls for each genotype. Scale bars in panels A-F are $5 \mu \mathrm{m}$. In A-F, dotted white circles are indicative of individual macrophage cell body based on 7-AAD staining of their regular nuclei and CRQ staining.

doi:10.1371/journal.pone.0031488.g006

phagocytosis of apoptotic cells. We also asked whether pkd2, rya-r44F and $r t p$ might genetically interact in phagocytosis. As anticipated, we found an interaction between $p k d 2$ and $r y a-r 44 F$, as heterozygous mutant macrophages for the $p k d 2$ knockout allele $a m o^{1}$ and the $r y a^{-}$ $r 44 F^{16}$ hypomorph allele were small and poorly engulfed apoptotic cells (figures 6C). These results are consistent with previous genetic interactions found between $p k d 2$ and $r y a-r 44 F$ in cell body wall contraction, and argue that $p k d 2$ acts in phagocytosis similarly to its role in body cell wall contraction by regulating $\mathrm{Ca}^{2+}$ homeostasis. We also observed a similar phenotype in double heterozygous mutant embryos for $a m o^{1}$ and the $r t p$ deficiency $D f(3 R) 3-4$, with smaller macrophages gathering around apoptotic corpses but engulfing poorly $($ figure $6 \mathbf{D})$ with a relative PI of $55.4 \pm 2.4 \%$ similar to that of amo ${ }^{1}$ homozygous macrophages $(\mathrm{p}>0.05)$ (figure 6G).

We previously linked RTP and Rya-r44F to the Dmel $\backslash$ Ced- 6 and DRPR pathway by showing that mutations of these genes all genetically interacted in vivo in phagocytosis of apoptotic cells, and by further demonstrating a role for $D m e \backslash \backslash C e d-6$ and $d r p r$ in SOCE in $\mathrm{S} 2$ cells [35]. Thus, we asked whether $p k d 2$ might also genetically interact with $d r p r$. We found that macrophages in double heterozygous for the $p k d 2$ knock-out allele $a m o^{1}$ and a null allele of $d r p r, \quad d r p r^{\text {rec } \Delta 5}[32,35]$ poorly engulf apoptotic cells (figure 6E), with a relative PI of $57.4 \pm 4.2 \%$ similar to that of amo $^{1}$ homozygous $(\mathrm{p}>0.05)$ (figure 6G). Macrophages of single heterozygotes $a m o^{1} /+$ or $d r p r^{r e c 45} /+$ had relative PIs of $82.1 \pm 3.8 \%$ and $101.02 \pm 8.3 \%$, respectively, similar to that of wild type macrophages (with $\mathrm{p}$ values $>0.05$ )(figure 6G). Thus, these results argue that $p k d 2$ and $d r p r$ are likely to act in a same pathway.

simu genetically interacts with $p k d 2$ but not with Dmellced-12

Our earlier results demonstrating a genetic interaction between the deficiencies, $D f(2 L)$ prd1.7 or $D f(2 L) P r l$ and $D f(2 L) b 87 e 25$, also led us to conclude that gene $X$ within the $D f(2 L)$ prd1.7 and $D f(2 L) p r l$ 
genomic regions and simu within the $D f(2 L) b 87 e 25$ region were likely to act in the same pathway. Having identified gene $X$ as $p k d 2$, we re-assessed the genetic interaction between the $p k d 2$ knockout allele $a m o^{1}$ and $D f(2 L) b 87 e 25$. As anticipated, macrophages in $a m o^{1} / D f(2 L) b 87 e 25$ heterozygous embryos were small and engulfed apoptotic cells as poorly as $a m o^{1}$ or $D f(2 L) b 87 e 25$ homozygous macrophages (figure 6F), with a PI of $49.2 \pm 4.1 \%$ versus $51.7 \pm 2.6 \%$ and $54.6 \pm 3 \%$, respectively (figure 6G)(with $\mathrm{p}$ values $>0.05)$. To further test whether $p k d 2$ genetically interacted with simu in this deficiency region, we next assessed the phagocytosis phenotype of macrophages in heterozygous embryos for $a m o^{1}$ and a null allele of simu, simu (a kind gift of Dr. E. Kurant [36]). We found their phagocytic ability to be reduced to a relative PI of $30.1 \pm 7.1 \%$ (figure $6 \mathbf{G}$ ), This PI is similar to that of drprec 45 and $D f(3 R) 3-4$ homozygous macrophages (figure 6G), and consistent with previous data reporting the engulfing capacity of $\operatorname{sim}^{2}$ homozygous mutants at about a third of that of wild-type macrophages [36]. This PI, however, is slightly lower than that of $a m o^{1}$ (PI of $51.7 \pm 2.6 \%, \mathrm{p}<0.05$ ) and $D f(2 L) b 87 e 25$ homozygous macrophages (PI of $54.6 \pm 3.0 \%$, $\mathrm{p}<0.001)$, as well as $a m o^{1} / D f(2 L) b 87 e 25$ heterozygous macrophages (PI of $49.2 \pm 4.1 \%, \mathrm{p}<0.001$ ) suggesting that the $D f(2 L) b 87 e 25$ deficiency may have slightly elevated levels of apoptosis or delete a phagocytosis suppressor. We previously reported that macrophages of $D f(3 R) 3-4$ heterozygotes were wild type for phagocytosis [35]. Here we further report that macrophages of single heterozygotes for either $a m o^{1}$ or simu ${ }^{2}$ had relative PIs of $82.1 \pm 8.3 \%$ and $95.4 \pm 3.8 \%$, respectively, similar to that of wild type macrophages (with $\mathrm{p}$ values $>0.05)$ (figure $6 \mathbf{G}$ ). Thus, together these results argue that $p k d 2$ genetically interacts with simu, although its role in apoptotic cell clearance appears less prominent than that of simu, drpr and rtp. Thus, PKD2 is likely to play a role in regulating $\mathrm{Ca}^{2+}$ homeostasis during phagocytosis, in concert with RTP, Rya$\mathrm{r} 44 \mathrm{~F}$, Dmel $\backslash$ Ced- 6 and DRPR. This is further supported by recent publications (since the first submission of this manuscript) reporting that the mammalian counterpart of PKD2, Polycystin2 (also known as PC2, which is encoded by the $p k d 2$ gene) can modulate $\mathrm{ER} \mathrm{Ca}^{2+}$ release and play a role in SOCE $[44,45]$.

Finally, our earlier results demonstrating a lack of genetic interaction between the $D f(2 L) e s c-P 3-0$ and $D f(2 L) b 87 e 25$ deficiencies also argued that Dmel\ced-12 and simu may not be genetically interacting and are likely to act in parallel or branched pathways. To further test this, we assessed the PI of heterozygotes macrophages for ced-12 $2^{19 F 3}$ and simu ${ }^{2}$. We found that both single heterogygous macrophages for $\operatorname{ced}-12^{19 F 3}$ and simu ${ }^{2}$, and their double heterozygous macrophages all had PIs similar to that of wild type (PIs of $96.1 \pm 5.8 \%, 95.4 \pm 3.8 \%$ and $89.6 \pm 5.8 \%$, respectively, with $\mathrm{p}$ values $>0.05$ when compared to wild type). Thus, like its C. elegans and mammalian counterparts, Dmel $\backslash$ Ced12 is likely to act in a parallel pathway to the SIMU/DRPR pathway.

\section{Discussion}

With the goal to pursue the genetic dissection of the molecular mechanisms underlying apoptotic cell clearance, we have characterized four Drosophila deficiency mutants with defects in this process, and have identified the homologue of C. elegans CED12, Dmel $\backslash$ Ced-12, as being required for phagocytosis. We propose that, as with its C. elegans counterpart, Dmel $\backslash$ Ced-12 is likely to act as a Guanidine Exchange Factor (GEF) for the small GTPase RAC2, which is also required for apoptotic cell clearance in the fly $(\boldsymbol{N C F}$, unpublished data $)$, and to promote actin cytoskeleton rearrangement during phagocytosis. As in $C$. elegans, our results further argue that Dmel $\backslash$ Ced- 12 also acts in a genetically distinct pathway to that of DRPR, the CED-1 homologue.

Our previous data demonstrated a role for RTP (UTA) in SOCE and apoptotic cell clearance, and linked RTP to the DRPR pathway [35]. Here, we have found a novel role for the TRPP-like cation channel, PKD2, in apoptotic cell clearance, and gathered evidence that it acts in the DRPR pathway, arguing that PKD2 is likely to also regulate $\mathrm{Ca}^{2+}$ homeostasis during phagocytosis. Calcium $\left(\mathrm{Ca}^{2+}\right)$ was shown to play a role in phagocytosis in mammalian cells, but its precise role in this process remains unclear and somewhat controversial. A rise in intracellular $\mathrm{Ca}^{2+}$ concentration $\left(\left[\mathrm{Ca}^{2+}\right] \mathrm{i}\right)$ in mammalian phagocytes was previously described during phagocytosis of various particles, but the molecular mechanisms underlying this rise in $\left[\mathrm{Ca}^{2+}\right] \mathrm{i}$ are poorly understood $[46,47,48]$. Thus our results on PKD2 provide us with a new component of the molecular machinery underlying $\mathrm{Ca}^{2+}$ homeostasis in phagocytosis.

At least one other Drosophila TRP channel, TRPML, was proposed to play a role in apoptotic cell clearance by macrophages and glial cells in a model for the neurodegenerative disorder known as Mucolipidosis type IV (MLIV), a lysosomal storage disorder with severe impairment in motor neurons [49]. In mammalian macrophages, a $\mathrm{Ca}^{2+}$-release activated $\mathrm{Ca}^{2+}$ (CRAC) channel activity and more recently a TRP channel activity were also detected, yet a role for store-operated $\mathrm{Ca}^{2+}$ Entry (SOCE) in controlling $\mathrm{Ca}^{2+}$ homeostasis during phagocytosis has remained controversial $[50,51,52]$. A TRP channel of the C family, TRPC1, has now been associated with SOCE in mammalian systems [53], and thus TRP channels may be part of a large multi-molecular complex that regulate $\mathrm{Ca}^{2+}$ homeostasis. Although evolutionary distant from PKD2, TRPC1 has been shown to participate in SOCE by physically interacting and forming a ternary complex with the ER $\mathrm{Ca}^{2+}$ sensor STIM1 and the CRAC channel ORAI1 [54]. Thus PKD2 may play a role in SOCE by physically interacting with dSTIM and dOrai and future studies will be required to test this hypothesis. Of note is that $\mathrm{PKD} 2$ possesses an ER retention domain, arguing that PKD2 might indeed play a role at the ER level rather than at the plasma membrane, however further studies will be required to assess its subcellular localization and function in $\mathrm{Ca}^{2+}$ homeostasis.

Like mammalian immunoreceptors, DRPR bears an immunoreceptor tyrosine-activation motif (ITAM) [33]. During axon pruning by Drosophila glial cells, the ITAM of DRPR is phosphorylated by Src-tyrosine family member, Src42A, which leads to the binding onto DRPR of Shark, a non-tyrosine kinase of the Syk and Zap-70 family [33]. The activity of such kinases can be $\mathrm{Ca}^{2+}$-dependent in mammalian cells $[55,56]$, as well as promote intracellular $\mathrm{Ca}^{2+}$ changes [57,58]. Thus, our working hypothesis is that an initial increase in $\left[\mathrm{Ca}^{2+}\right]$ i either via release of $\mathrm{Ca}^{2+}$ from the ER and/or via SOCE activates Src42A and DRPR phosphorylation thereby promoting the recruitment of Dmel $\backslash$ Ced-6 and/or Shark to DRPR. More recently, in mouse macrophages, the TRPV2 channel was shown to play a crucial role in particle recognition and phagocytosis by macrophages, where it promotes immunoreceptor clustering by inducing membrane depolarization and phospholipiddependent actin depolymerization [59]. Thus PKD2 and/or TRPML may play similar roles in Drosophila macrophages and future studies will be necessary to determine the precise role of TRP channels in these cells in the context of apoptotic cells and bacteria clearance, as well as their precise role in calcium homeostasis during this process. 


\section{Methods}

\section{Fly strains}

All fly stocks were obtained from the Szeged or Bloomington Drosophila stock centres, unless otherwise specified. Whenever possible, fly strains were crossed to a balancer chromosome containing a kruppel::GFP (kr-GFP) or twist::GFP (twi-GFP) reporter transgene and homozygous mutant embryos were selected against $k r$-GFP expression on a Leica fluorescent stereo-microscope equipped with GFP filter (Leica Microsystems Inc, UK), or detected by their lack of GFP immunoreactivity by confocal microscopy (Bio-Rad Radiance confocal equipped with a Nikon upright microscope). crq-Gal4, UAS-eGFP transgenic flies were a generous gift from Hervé Agaisse (Yale University) and Norbert Perrimon (Harvard Medical School). We generated UAStransgenic flies by injecting yw embryos with a mixture of $U A S$ construct and helper plasmid at the concentration of $200 \mathrm{ng}$ and $100 \mathrm{ng} / \mu \mathrm{l}$ respectively following standard procedures. Fly stocks were all maintained at $25^{\circ} \mathrm{C}$ in LMS600 incubators with 12 hourlight cycles (unless otherwise stated) on standard fly medium.

\section{Acridine orange $(\mathrm{AO})$}

Embryos were collected for two hours at $25^{\circ} \mathrm{C}$ on juice plates and aged for $17 \mathrm{hrs}$ at $18^{\circ} \mathrm{C}$ to stage 13 . Embryos were treated with $50 \%$ bleach to remove their chorion, and washed extensively in bi-distilled water. Acridine orange (AO) staining was performed as described previously by shaking the embryo for seven minutes in an equal mixture of heptane and PBS containing $5 \mu \mathrm{g} / \mathrm{ml}$ of AO [34]. Stained embryos were transferred onto a glass slide, covered with halocarbon oil 700 and a coverslip, and subsequently viewed within 20 minutes under a Leica fluorescent MZ FL III stereo-microscope equipped with both green and red filters (Leica Microsystems Inc, UK).

\section{Immunostainings}

Appropriately staged embryos were fixed in 4\% formaldehyde/ PBS (electron microscopy grade, TAAB), devitellinized with a $1: 1$ volume mixture of methanol/n-Heptane, re-hydrated through a series of ethanol/PBT $(25 \% / 75 \%, 50 \% / 50 \%$ and $25 \% / 75 \%$, $100 \%$ ), blocked for $1 \mathrm{hr}$ at room temperature in PBT containing $10 \%$ bovine serum albumin (BSA), and stained with either the CRQ Ab (IgG purified rabbit polyclonal antibody) alone, as previously described in [28] or using both the anti-CRQ and antiGFP antibodies (mouse monoclonal antibody, Gibco) at 1:1,000 and 1:4,000 dilutions respectively in PBT containing $1 \% \mathrm{BSA}$ at $4^{\circ} \mathrm{C}$ overnight. Three washes of 30 minutes in PBT were performed at room temperature and followed by $1 \mathrm{hr}$ of incubation in PBT containing 1\%BSA with appropriate fluorescent secondary antibodies. Anti-rabbit and anti-mouse fluoresceincoupled secondary antibodies were from Vector laboratories and used at a 1:1,000 dilution. The anti-rabbit Cy5 antibody (Jackson Laboratories) was used at a 1:1,000 dilution. After three washes for 30 minutes in PBT and two quick washes in PBS, a 7-amino actinomycin D (7-AAD) staining was performed in PBS at a concentration of $5 \mathrm{mg} / \mathrm{ml}$, followed by three washes in PBS for 510 minutes [29]. Stained embryos were mounted in vectashield medium (Vector laboratories). Embryos were observed on a BioRad Radiance confocal microscope equipped with a Nikon upright microscope using $25 \times, 40 \times, 60 \times$, and/or $100 \times$ objectives. Images were collected using the lasersharp 2000 software and further processed using versions 4.0 or 6.0 of Adobe Photoshop software or Image J $1.34 \mathrm{~g}(\mathrm{NIH})$. For the VASA antibody staining, the VASA primary antibody made in chicken was diluted at 1:10,000 in PBT, followed by detection with an anti- chicken secondary antibody coupled to FITC diluted at 1:1,000 in PBT.

\section{Terminal deoxyribonucleotide transferase (TdT)- mediated dUTP Nick End Labelling (TUNEL)}

Embryos were collected, staged, fixed and rehydrated as described above for immunostaining. Once in PBT, the embryos were treated with $10 \mu \mathrm{g} / \mathrm{ml}$ of Proteinase K in PBS for 4 minutes, followed by two washes of 5 minutes in PBT. The embryos were post-fixed in $4 \%$ paraformaldehyde (EM grade) in PBS for 20 minutes at room temperature on a rotating platform. After five washes for 5 minutes in PBT, the embryos were incubated for 1 hour at room temperature in equilibration buffer of the apoptag kit (Chemicon), followed by an overnight incubation at $37^{\circ} \mathrm{C}$ in $110 \mu \mathrm{l}$ of reaction buffer containing 2 volumes of $\mathrm{TdT}$ and 1 volume of $0.3 \%$ Trito-X100, while shaking. Embryos are then incubated in $1 \mathrm{ml}$ of Stop buffer for 3 hours at $37^{\circ} \mathrm{C}$, while shaking, washed three times in PBT for 5 minutes, blocked in PBT containing $5 \%$ of Normal Goat Serum (NGS) and $2 \mathrm{mg} / \mathrm{ml}$ of BSA for 1 hour at room temperature. The incorporation of digoxygenin dUTP into the nicked-end of apoptotic cells' DNA is then detected using a FITC-coupled digoxygenin primary antibody (Roche) used at a 1:100 dilution in fresh blocking buffer for 1 hour at room temperature. After three washes in PBT of 20 minutes each, the embryos are mounted between slides and coverslips in vectashield mounting medium (Vector), and observed using a Leica SP5 confocal microscope.

\section{Single embryo polymerase chain reactions (PCR)}

Mapping of the deletions by PCRs were performed as previously described in [29] using template DNA extracted from homozygous embryos that were manually sorted against Kr-GFP expression. Primers used are listed in the Supplementary Text S1. In all reactions, a RP49 primer set was used as an internal control for the presence of DNA. RP49_S: 5' ATACAGGCGCAAGATCGTGA 3', RP49_AS: 5' GTGTATTCGGACCACGTTACA $3^{\prime}$. PCR cycles were as follows: $94^{\circ} \mathrm{C}$ for $5 \mathrm{~min}$ for one cycle, denaturation at $94^{\circ} \mathrm{C}$ for $1 \mathrm{~min}$, annealing at $65^{\circ} \mathrm{C}$ for $1 \mathrm{~min}$, extension at $72^{\circ} \mathrm{C}$ for $1 \mathrm{~min} 15 \mathrm{sec}$, for $30 \mathrm{cycles}$, and final extension at $72^{\circ} \mathrm{C}$ for $10 \mathrm{~min} .15 \mu \mathrm{l}$ of each PCR reaction were loaded onto a $2 \%$ agarose/TBE (Tris/Borate/EDTA) gel and their products were separated, visualized and photographed on a Syngene genelink trans-illuminating system.

\section{Plasmid construct}

The RE62284 EST containing the cDNA sequence of Dmel $\backslash$ ced12 (Berkeley Drosophila Genome Project (BDGP)) was obtained from the Berkeley Drosophila Genome Project (BDGP) collection library. The Dmel $\backslash e d-12 \mathrm{cDNA}$ insert was excised from the $p F L C 1$ vector following a triple restriction digests using 5 Units of the $K p n I, \mathcal{N} o t I$, and BsphI enzymes and appropriate buffer in the presence of $0.1 \mathrm{mg} / \mathrm{ml}$ of BSA. The KpnI/NotI cDNA insert was purified by gel extraction and ligated into the KpnI/NotI-digested pUAST vector using 5 Units of T4 DNA ligase in a $20 \mu \mathrm{l}$ final volume overnight at room temperature. $1 \mu \mathrm{l}$ of ligation was electroporated into DH5alpha bacteria from Invitrogen using a Bio-Rad electroporator and following manufacturer's instructions. Transformed bacteria were grown in $1 \mathrm{ml}$ of SOC medium (Gibco) in a bacterial shaker at $37^{\circ} \mathrm{C}$, and subsequently plated onto LB agar plates containing $100 \mu \mathrm{g} / \mathrm{ml}$ of ampicillin and incubated overnight at $37^{\circ} \mathrm{C}$. Single colonies were picked and grown into $2 \mathrm{ml}$ of LB medium containing $50 \mu \mathrm{g} / \mathrm{ml}$ of ampicillin. Preparations of plasmid DNA were performed using the Qiagen 
mini-prep kit. Individual clones containing the insert were identified by restriction map analysis and gel electrophoresis. Restriction digests were all conducted for one hour at $37^{\circ} \mathrm{C}$ and made use of $5 \mu \mathrm{l}$ of each plasmid preparations and 5 Units of appropriate restriction enzymes in $20 \mu \mathrm{l}$ final volume containing $10 \%$ volume of appropriate restriction buffer and $0.1 \mathrm{mg} / \mathrm{ml}$ of BSA.

\section{In vivo rescue experiment}

To re-express Dmel $\backslash$ ced-12in macrophages in the $D f(2 L)$ prd1.7 background, the following three stocks were established: (1) $y$, w, UAS-dCed-12; Df(2L)prd1.7 /CyO, (2) w; Df(2L)prd1.7/ CyO; crqGal4, UAS-eGFP and (3) w, crq-gal4; Df(2L)prd1.7/CyO. The crq-gal4 drivers were a kind gift of $\mathrm{H}$. Agaisse and N. Perrimon, Harvard Medical School. The use of the crq-gal4, UAS-eGFP chromosome allows us to detect macrophages expressing cytoplasmic eGFP under the control of the crq-gal4 driver. Females of the deficiency line carrying the UAS-Dme $\backslash$ ced-12 transgene were crossed to males of the deficiency line carrying the crq-gal4 driver either on the $\mathrm{X}$ or on the third chromosome. Homozygous embryos were sorted for their segmentation defect on the confocal microscope prior to their analysis. All such embryos obtained with the crq-gal4 on the third chromosome were rescued as they carried both one copy of the transgene and one copy of the driver. By contrast, when using the crq-galt driver on the $\mathrm{X}$ chromosome, only half of the homozygous deficient embryos (the females) were carrying both the driver and the transgene on the $\mathrm{X}$ chromosome, as expected by Mendel's law. The fly line carrying the genomic rescue transgene of prd in the background of the $D f(2 L) p r d 1.7$ deficiency was a kind gift of Drs. Eric Frei and Marcus Noll (Institute for Molecular Biology, University of Zurich).

\section{Generation of Germ Line Clones (GLCs)}

The dced-12[19F3], dced-12[PB] and dced-12[8c6] mutations were recombined onto the FLP recombination target (FRT)-containing second chromosome of $w ; a[1] d p$ ov[1] b[1] $\operatorname{pr}[1] P\{y[+t 7.2]=-$ neoFRT \} $40 A$ (Bloomington Stock Center) and crossed to males of a $h s-F L P, P\{y[+t 7.2=n e o F R T\} 40 A, U b i-G F P / C y O$ stock. The progeny from this cross was heat-shocked at $37 \mathrm{C}$ for 1 hour, three times with 24 hours of recovery between each heat-shock, starting at late L2 or L3 stages (i.e. on days 4, 5 and 6 of culture). Virgin females $h s-F L P, P\{r y[+t 7.2=n e o F R T\} 40 A, U b i-G F P / d c e d-12[19 F 3]$, or $/ d c e d-12[P B]$, or $/ d c e d-12[8 c 6]$ were recovered and crossed to heterozygous males $D f(2 \mathrm{~L}) \mathrm{prd1.7/CyO}, \mathrm{kr}$-GFP or $\mathrm{Df}(2 \mathrm{~L}) \mathrm{prl} / \mathrm{Cy} \mathrm{O}, \mathrm{kr}$ GFP. The phagocytosis phenotype of embryos resulting from this cross and sorted against GFP expression prior to fixation was assessed as described above. Of note is that no viable stage 13 embryos could be recovered when using the dced-12[19F3] allele.

\section{Statistical analyses}

Phagocytic indexes (PIs) presented in this study are average PIs calculated from confocal images taken on five to 15 different embryos per genotype, in which confocal image-stacks of 12 to 24 sections through macrophages per embryo were taken at $60 \times$ and

\section{References}

1. Jacobson MD, Weil M, Raff MC (1997) Programmed cell death in animal development. Cell 88: 347-354.

2. Raff MC, Barres BA, Burne JF, Coles HS, Ishizaki Y, et al. (1994) Programmed cell death and the control of cell survival. Philos Trans R Soc Lond B Biol Sci 345: 265-268.

3. Wyllie AH, Kerr JF, Currie AR (1980) Cell death: the significance of apoptosis. Int Rev Cytol 68: 251-306.

4. deCathelineau AM, Henson PM (2003) The final step in programmed cell death: phagocytes carry apoptotic cells to the grave. Essays Biochem 39: 105-117. a zoom of 1.7. Standard errors of the mean (SEMs) were derived from the PIs calculated from all embryos per genotype. One wayanalyses of variance (one way-ANOVAs) were performed to compare PIs between embryos of various genotypes that returned $\mathrm{p}$ values, which can be found throughout the text.

\section{Supporting Information}

Figure S1 Characterization of macrophage migration phenotypes in deficient and $\operatorname{prd}^{\boldsymbol{s}}$ homozygous mutant embryos. In A-F embryos were aged to stage 13, fixed, their macrophages immunostained with the CRQ Ab (green) and apoptotic corpses detected with 7-AAD staining (red). Confocal images of twelve focal plans taken through the head of a wild-type $y, w$ embryo (A and $\mathbf{D}), D f(2 L) \operatorname{prd1.7}(\mathbf{B})$ and $D f(2 L) \operatorname{Prl}(\mathbf{C})$, $D f(2 L) E s c-P 3-0(\mathbf{E})$ and $p r d^{o}(\mathbf{F})$ homozygous mutant embryos. In all embryos, macrophages properly migrate throughout the head of the embryo surrounding the brain lobes, and appear large as they engulf multiple apoptotic cells. Scale bars are $50 \mu \mathrm{m}$.

(TIF)

Figure S2 Characterization of macrophage phagocytosis phenotypes in prd $^{\boldsymbol{s}}$ homozygous mutant embryos. In A-B embryos were aged to stage 13, fixed, their macrophages immunostained with the CRQ Ab (green) and apoptotic corpses detected with 7-AAD staining (red). Confocal images of twelve focal plans were taken through the head of a wild-type $y$, w embryo (A) and $\operatorname{prd}^{8}$ (B) homozygous mutant embryos. Macrophages in $\operatorname{prd}^{\circ}$ appear larger as they engulf more apoptotic cells compared to wild-type macrophages. Scale bars are $10 \mu \mathrm{m}$. In A-B, dotted white circles are indicative of individual macrophage cell bodies based on 7-AAD staining of their regular nuclei and CRQ staining.

(TIF)

Text S1 Additional Material. This file lists the primers used to map the breakpoints of the deficiencies used in the present study (see Material and Methods).

(DOC)

\section{Acknowledgments}

We wish to thank Hervé Agaisse and Norbert Perrimon for the crq-Gal4, UAS-eGFP flies, Erika Geisbrecht and Susan Abmayr for the Dmel $\backslash$ ced-12null alleles, Eric Frei and Marcus Noll for the prd genomic rescue fly line, the Bloomington and Szeged Drosophila stock centres for all deficiency fly stocks used in this study. We thank Andrew Vaughan for his help with confocal microscopy. This is manuscript \#21278 from The Scripps Research Institute.

\section{Author Contributions}

Conceived and designed the experiments: EVG NGF. Performed the experiments: EVG ES HX NCF. Analyzed the data: EVG ES HX NCF. Wrote the paper: EVG NCF. Provided feedback on the manuscript: ES HX.

5. Savill J, Gregory C, Haslett C (2003) Cell biology. Eat me or die. Science 302: 1516-1517.

6. Hauwel M, Furon E, Canova C, Griffiths M, Neal J, et al. (2005) Innate (inherent) control of brain infection, brain inflammation and brain repair: the role of microglia, astrocytes, "protective" glial stem cells and stromal ependymal cells. Brain Res Brain Res Rev 48: 220-233.

7. Franc NC (2002) Phagocytosis of apoptotic cells in mammals, caenorhabditis elegans and Drosophila melanogaster: molecular mechanisms and physiological consequences. Front Biosci 7: d1298-1313. 
8. Cohen PL, Caricchio R, Abraham V, Camenisch TD, Jennette JC, et al. (2002) Delayed apoptotic cell clearance and lupus-like autoimmunity in mice lacking the c-mer membrane tyrosine kinase. J Exp Med 196: 135-140.

9. Gaipl US, Sheriff A, Franz S, Munoz LE, Voll RE, et al. (2006) Inefficient clearance of dying cells and autoreactivity. Curr Top Microbiol Immunol 305: 161-176.

10. Pittoni V, Valesini G (2002) The clearance of apoptotic cells: implications for autoimmunity. Autoimmun Rev 1: 154-161.

11. Watson S, Cailhier JF, Hughes J, Savill J (2006) Apoptosis and glomerulonephritis. Curr Dir Autoimmun 9: 188-204.

12. Yamaguchi H, Fujimoto T, Nakamura S, Ohmura K, Mimori T, et al. (2010) Aberrant splicing of the milk fat globule-EGF factor 8 (MFG-E8) gene in human systemic lupus erythematosus. European journal of immunology 40: 1778-1785.

13. Yamaguchi H, Takagi J, Miyamae T, Yokota S, Fujimoto T, et al. (2008) Milk fat globule EGF factor 8 in the serum of human patients of systemic lupus erythematosus. Journal of leukocyte biology 83: 1300-1307.

14. Kinchen JM, Cabello J, Klingele D, Wong K, Feichtinger R, et al. (2005) Two pathways converge at CED-10 to mediate actin rearrangement and corpse removal in C. elegans. Nature 434: 93-99.

15. Mangahas PM, Zhou Z (2005) Clearance of apoptotic cells in Caenorhabditis elegans. Semin Cell Dev Biol 16: 295-306.

16. Ellis RE, Jacobson DM, Horvitz HR (1991) Genes required for the engulfment of cell corpses during programmed cell death in Caenorhabditis elegans. Genetics 129: 79-94.

17. Zhou Z, Hartwieg E, Horvitz HR (2001) CED-1 is a transmembrane receptor that mediates cell corpse engulfment in C. elegans. Cell 104: 43-56.

18. Wu YC, Horvitz HR (1998) The C. elegans cell corpse engulfment gene ced-7 encodes a protein similar to ABC transporters. Cell 93: 951-960.

19. Smits E, Van Criekinge W, Plaetinck G, Bogaert T (1999) The human homologue of Caenorhabditis elegans CED-6 specifically promotes phagocytosis of apoptotic cells. Curr Biol 9: 1351-1354.

20. Reddien PW, Horvitz HR (2000) CED-2/CrkII and CED-10/Rac control phagocytosis and cell migration in Caenorhabditis elegans. Nat Cell Biol 2: 131-136.

21. Wu YG, Horvitz HR (1998) C. elegans phagocytosis and cell-migration protein CED-5 is similar to human DOCK180. Nature 392: 501-504.

22. Wu YC, Tsai MC, Cheng LC, Chou CJ, Weng NY (2001) C. elegans CED-12 acts in the conserved crkII/DOCK180/Rac pathway to control cell migration and cell corpse engulfment. Dev Cell 1: 491-502.

23. Zhou Z, Caron E, Hartwieg E, Hall A, Horvitz HR (2001) The C. elegans PH domain protein CED-12 regulates cytoskeletal reorganization via a Rho/Rac GTPase signaling pathway. Dev Cell 1: 477-489.

24. Gumienny TL, Brugnera E, Tosello-Trampont AC, Kinchen JM, Haney LB, et al. (2001) CED-12/ELMO, a novel member of the CrkII/Dock180/Rac pathway, is required for phagocytosis and cell migration. Cell 107: 27-41.

25. Pazdera TM, Janardhan P, Minden JS (1998) Patterned epidermal cell death in wild-type and segment polarity mutant Drosophila embryos. Development 125 : 3427-3436.

26. Sonnenfeld MJ, Jacobs JR (1995) Macrophages and glia participate in the removal of apoptotic neurons from the Drosophila embryonic nervous system. J Comp Neurol 359: 644-652.

27. Tepass U, Fessler LI, Aziz A, Hartenstein V (1994) Embryonic origin of hemocytes and their relationship to cell death in Drosophila. Development 120: 1829-1837.

28. Franc NC, Dimarcq JL, Lagueux M, Hoffmann J, Ezekowitz RA (1996) Croquemort, a novel Drosophila hemocyte/macrophage receptor that recognizes apoptotic cells. Immunity 4: 431-443.

29. Franc NC, Heitzler P, Ezekowitz RA, White K (1999) Requirement for croquemort in phagocytosis of apoptotic cells in Drosophila. Science 284: 1991-1994.

30. Manaka J, Kuraishi T, Shiratsuchi A, Nakai Y, Higashida H, et al. (2004) Draper-mediated and phosphatidylserine-independent phagocytosis of apoptotic cells by Drosophila hemocytes/macrophages. The Journal of biological chemistry 279: 48466-48476.

31. Awasaki T, Tatsumi R, Takahashi K, Arai K, Nakanishi Y, et al. (2006) Essential role of the apoptotic cell engulfment genes draper and ced-6 in programmed axon pruning during Drosophila metamorphosis. Neuron 50: 855-867.

32. Freeman MR, Delrow J, Kim J, Johnson E, Doe CQ (2003) Unwrapping glial biology: Gcm target genes regulating glial development, diversification, and function. Neuron 38: 567-580.

33. Ziegenfuss JS, Biswas R, Avery MA, Hong K, Sheehan AE, et al. (2008) Draperdependent glial phagocytic activity is mediated by Src and Syk family kinase signalling. Nature 453: 935-939.

34. Silva E, Au-Yeung HW, Van Goethem E, Burden J, Franc NC (2007) Requirement for a Drosophila E3-ubiquitin ligase in phagocytosis of apoptotic cells. Immunity 27: 585-596.

35. Cuttell L, Vaughan A, Silva E, Escaron CJ, Lavine M, et al. (2008) Undertaker, a Drosophila Junctophilin, links Draper-mediated phagocytosis and calcium homeostasis. Cell 135: 524-534
36. Kurant E, Axelrod S, Leaman D, Gaul U (2008) Six-microns-under acts upstream of Draper in the glial phagocytosis of apoptotic neurons. Cell 133: 498-509.

37. Abrams JM, White K, Fessler LI, Steller H (1993) Programmed cell death during Drosophila embryogenesis. Development 117: 29-43.

38. Frigerio G, Burri M, Bopp D, Baumgartner S, Noll M (1986) Structure of the segmentation gene paired and the Drosophila PRD gene set as part of a gene network. Cell 47: 735-746.

39. Geisbrecht ER, Haralalka S, Swanson SK, Florens L, Washburn MP, et al. (2008) Drosophila ELMO/CED-12 interacts with Myoblast city to direct myoblast fusion and ommatidial organization. Developmental biology 314: 137-149.

40. Bianco A, Poukkula M, Cliffe A, Mathieu J, Luque CM, et al. (2007) Two distinct modes of guidance signalling during collective migration of border cells. Nature 448: 362-365.

41. Gao Z, Joseph E, Ruden DM, Lu X (2004) Drosophila Pkd2 is haploidinsufficient for mediating optimal smooth muscle contractility. The Journal of biological chemistry 279: 14225-14231.

42. Gao Z, Ruden DM, Lu X (2003) PKD2 cation channel is required for directional sperm movement and male fertility. Current biology : CB 13: 2175-2178.

43. Watnick TJ, Jin Y, Matunis E, Kernan MJ, Montell C (2003) A flagellar polycystin-2 homolog required for male fertility in Drosophila. Current biology : CB 13: 2179-2184.

44. Santoso NG, Cebotaru L, Guggino WB (2011) Polycystin-1, 2, and STIM1 interact with $\mathrm{IP}(3) \mathrm{R}$ to modulate ER Ca release through the PI3K/Akt pathway. Cellular physiology and biochemistry : international journal of experimental cellular physiology, biochemistry, and pharmacology 27: 715-726.

45. Spirli C, Locatelli L, Fiorotto R, Morell CM, Fabris L, et al. (2011) Altered store operated calcium entry increases cAMP production and ERK1/2 phosphorylation in Polycystin-2 defective cholangiocytes. Hepatology.

46. Dewitt S, Hallett MB (2002) Cytosolic free $\mathrm{Ca}(2+)$ changes and calpain activation are required for beta integrin-accelerated phagocytosis by human neutrophils. The Journal of cell biology 159: 181-189.

47. Rubartelli A, Poggi A, Zocchi MR (1997) The selective engulfment of apoptotic bodies by dendritic cells is mediated by the alpha(v)beta 3 integrin and requires intracellular and extracellular calcium. European journal of immunology 27: 1893-1900.

48. Tejle K, Magnusson KE, Rasmusson B (2002) Phagocytosis and phagosome maturation are regulated by calcium in $\mathrm{J} 774$ macrophages interacting with unopsonized prey. Bioscience reports 22: 529-540.

49. Venkatachalam K, Long AA, Elsaesser R, Nikolaeva D, Broadie K, et al. (2008) Motor deficit in a Drosophila model of mucolipidosis type IV due to defective clearance of apoptotic cells. Cell 135: 838-851.

50. Greenberg S, el Khoury J, di Virgilio F, Kaplan EM, Silverstein SC (1991) $\mathrm{Ca}(2+)$-independent $\mathrm{F}$-actin assembly and disassembly during $\mathrm{Fc}$ receptormediated phagocytosis in mouse macrophages. The Journal of cell biology 113: 757-767.

51. Hishikawa T, Cheung JY, Yelamarty RV, Knutson DW (1991) Calcium transients during $\mathrm{F}_{\mathrm{C}}$ receptor-mediated and nonspecific phagocytosis by murine peritoneal macrophages. The Journal of cell biology 115: 59-66.

52. McNeil PL, Swanson JA, Wright SD, Silverstein SC, Taylor DL (1986) Fcreceptor-mediated phagocytosis occurs in macrophages without an increase in average [Ca++]i. The Journal of cell biology 102: 1586-1592.

53. Ambudkar IS, Ong HL, Liu X, Bandyopadhyay BC, Cheng KT (2007) TRPC1: the link between functionally distinct store-operated calcium channels. Cell calcium 42: 213-223.

54. Ong HL, Cheng KT, Liu X, Bandyopadhyay BC, Paria BC, et al. (2007) Dynamic assembly of TRPC1-STIM1-Orail ternary complex is involved in store-operated calcium influx. Evidence for similarities in store-operated and calcium release-activated calcium channel components. The Journal of biological chemistry 282: 9105-9116.

55. Papp S, Fadel MP, Kim H, McCulloch CA, Opas M (2007) Calreticulin affects fibronectin-based cell-substratum adhesion via the regulation of c-Src activity. The Journal of biological chemistry 282: 16585-16598.

56. Wang X, Sada K, Yanagi S, Yang C, Rezaul K, et al. (1994) Intracellular calcium dependent activation of p72syk in platelets. Journal of biochemistry 116 : 858-861.

57. Briddon SJ, Melford SK, Turner M, Tybulewicz V, Watson SP (1999) Collagen mediates changes in intracellular calcium in primary mouse megakaryocytes through syk-dependent and -independent pathways. Blood 93: 3847-3855.

58. Melford SK, Turner M, Briddon SJ, Tybulewicz VL, Watson SP (1997) Syk and Fyn are required by mouse megakaryocytes for the rise in intracellular calcium induced by a collagen-related peptide. The Journal of biological chemistry 272: 27539-27542.

59. Link TM, Park U, Vonakis BM, Raben DM, Soloski MJ, et al. (2010) TRPV2 has a pivotal role in macrophage particle binding and phagocytosis. Nature immunology 11: 232-239. 